\title{
Small Angle Scattering by Dislocations
}

Robb Thomson, Emeritus

L. E. Levine

G. G. Long

U.S. DEPARTMENT OF COMMERCE

Technology Administration

National Institute of Standards

and Technology

Gaithersburg, MD 20899-0001

QC 



\section{NISTIR 6117}

\section{Small Angle Scattering by Dislocations}

Robb Thomson, Emeritus

L. E. Levine

G. G. Long

U.S. DEPARTMENT OF COMMERCE

Technology Administration

National Institute of Standards

and Technology

Gaithersburg, MD 20899-0001

March 9, 1998

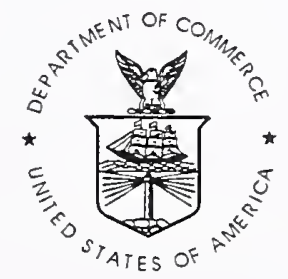

U.S. DEPARTMENT OF COMMERCE

William M. Daley, Secretary

TECHNOLOGY ADMINISTRATION

Gary R. Bachula, Acting Under Secretary

for Technology

NATIONAL INSTITUTE OF STANDARDS

AND TECHNOLOGY

Raymond G. Kammer, Director 



\title{
Small Angle Scattering by Dislocations
}

\author{
Robb Thomson, Emeritus, L. E. Levine, G. G. Long \\ NIST, Gaithersburg 20899, MD
}

(March 9, 1998)

\begin{abstract}
It is shown that the small-angle scattering of X-rays or neutrons by dislocations within a deformed metal, which are partially ordered into wall-like structures, is characterized by several factors. Principally these are associated with: 1) a single dislocation or dipole, 2) the dislocation configuration in the plane of the wall, and 3) the distribution of dislocations across the wall thickness. With the assumption of isotropic elasticity, small-angle scattering will be sensitive only to the edge components of the dislocations. The scattered intensity is dominated by scattering from dislocations which lie perpendicular to the scattering vector, $\overrightarrow{\mathbf{q}}$, and reaches a maximum when $\overrightarrow{\mathbf{q}}$ is normal to the slip plane of these dislocations. Above a particular $|\overrightarrow{\mathbf{q}}|$, the scattered intensity is sensitive only to the total edge dislocation content of the visible dislocations (i.e. scattering is incoherent), while below this value, the scattering is dominated by how the dislocations are distributed in walls. For walls normal to their slip planes, the configuration factor will reflect the dislocation distribution in the plane of the wall, while for walls parallel to their slip planes, the distribution in the thickness direction will be visible. Therefore, even though a deformed material is composed of complicated dislocation structures, only those segments conforming to these rather strict prescriptions will be singled out for scattering, and by adjusting the beam/slip system geometry, many parameters of the microstructure can be determined experimentally.
\end{abstract}




\section{INTRODUCTION}

This paper is motivated by experiments ${ }^{1}$ being carried out on the NIST materials science beam line at the National Synchrotron Light Source at Brookhaven National Laboratory, exploring ultra-small-angle X-ray scattering (USAXS) by deformed single-crystal metals. Here, we consider the theory of small-angle scattering from individual defects (dislocations and dislocation dipoles) and present a framework for understanding the role of wall configurations composed of these defects.

The mechanical response of a metal to plastic deformation has traditionally been divided into several stages. Stage I is characterized by a nearly constant flow stress as a function of applied strain and is only observed in single crystals at temperatures much lower than their melting points. In stage II, the stress/strain behavior becomes linear with a significant slope, resulting in work hardening. This stage II slope is temperature independent. In stage III, temperature-dependent recovery processes occur resulting in a decreasing, near-parabolic work hardening. The existence of later stages of work hardening have also been reported, but stages II and III are the most important industrially and discussion is restricted to these stages.

According to the current, imperfect, understanding of deformation in fcc metals, the elastic self energy of the system drives the dislocations to organize themselves into partiallyordered wall structures. In stage $\mathrm{II}^{2-6}$, where our interest begins, dislocation walls often develop parallel to the active slip planes, perhaps forming partially-ordered Taylor lattices ${ }^{2}$. These wall structures are sometimes referred to as "carpets". In Stage III, the walls form the boundaries of space-filling cells ${ }^{2-6}$. There have also been proposals,${ }^{7,8}$ partially supported by observations, that the walls may include dislocation dipoles. Thus, we will focus on scattering by dislocations distributed in walls of various types.

The theory of small-angle scattering by dislocations has been the subject of a number of earlier papers ${ }^{9-12}$, stimulated by early small-angle scattering experiments with X-rays (SAXS) and neutrons (SANS). However, the importance of the wall and cellular structures described above was not fully appreciated at the time of this work, so the theory was necessarily incomplete. Seeger and Kroner ${ }^{9}$ carried out an elegant analysis for the dilatation field of the edge dislocation for the general case in which the dislocation is not straight. However, since we will be working with mostly $2 \mathrm{D}$ wall structures, we find the $2 \mathrm{D}$ theory of Atkinson and Hirsch ${ }^{12}$ more in tune with our point of view, and we will refer often to it. Certainly dislocations in deformed metals are not straight. Nevertheless, 2D theory is appropriate for this problem because the dislocations roughly approximate straight lines over the length scales probed by the small-angle scattering experiments. In terms of the theory presented in this paper, it is necessary for the dislocations to be approximately straight over length scales much greater than the inter-dislocation distance.

In contrast to Bragg peak broadening ${ }^{13}$, small-angle scattering is caused exclusively by the local volume change induced by the dislocations. Thus, in the linear elastic approximation, only the edge components of dislocations will be visible. This is because screw dislocations have no net volume change. We focus on the elastic part of the field because the additional volume changes due to nonlinearity in the strain fields of both edges and screws can be neglected in practice ${ }^{12}$ for SAXS.

In the kinematic or single-scattering approximation, the scattered intensity is composed 
of a product of several factors: a factor for the individual atoms, one for a single defect (either single dislocation or dislocation dipole), a factor associated with a configuration of identical defects, and, finally, a form factor for the entire sample. In the following sections, we address each of these in turn, except for the atomic factor, which in the case of a pure metal can not provide an additional scattering contrast mechanism, and the sample size factor, which appears in a scattering vector range totally unreachable in practice. The analysis sections are followed by a section on computer simulations of relevant dislocation structures to validate the analysis.

\section{SINGLE DEFECT STRUCTURE FACTORS}

The scattering at small angles can be written in terms of the total scattering amplitude function, $A$, as ${ }^{14}$

$$
\begin{aligned}
I & =A(\overrightarrow{\mathbf{q}}) A^{*}(\overrightarrow{\mathbf{q}}), \\
A(\overrightarrow{\mathbf{q}}) & =a_{a} \int\left(1+\phi_{t o t}(\overrightarrow{\mathbf{x}})\right) \exp (i \overrightarrow{\mathbf{q}} \cdot \overrightarrow{\mathbf{x}}) d \overrightarrow{\mathbf{x}},
\end{aligned}
$$

where $a_{a}$ is the scattering amplitude for a unit volume of perfect crystal, and the integral is the amplitude structure factor for the scattering volume. The dislocations within this volume contribute a total fractional density change, $\phi_{t o t}$, at the point $\overrightarrow{\mathbf{x}}$. $\overrightarrow{\mathbf{q}}$ is the scattering vector. The first term in the integrand is scattering from the entire sample which only appears at extremely low $|\overrightarrow{\mathbf{q}}|$. It does not contribute to the visible small-angle scattering and can be ignored. Thus, the scattering amplitude becomes

$$
A(\overrightarrow{\mathbf{q}})=a_{a} \int \phi_{t o t}(\overrightarrow{\mathbf{x}}) \exp (i \overrightarrow{\mathbf{q}} \cdot \overrightarrow{\mathbf{x}}) d \overrightarrow{\mathbf{x}} .
$$

If the defects are distributed over the volume of the material at the positions, $\overrightarrow{\mathbf{R}}_{i}$, then the fractional density change, $\phi_{t o t}(\overrightarrow{\mathbf{x}})$ is the linear sum of the contributions from each defect, and if they are all equivalent, then a decomposition can be made,

$$
\mathcal{I}=\frac{I}{\left|a_{a}\right|^{2}}=\left|a_{d}\right|^{2} c
$$

where

$$
\begin{aligned}
a_{d} & =\int \phi(\overrightarrow{\mathbf{x}}) \exp \{i \overrightarrow{\mathbf{q}} \cdot \overrightarrow{\mathbf{x}}\} d \overrightarrow{\mathbf{x}} \\
c & =\sum_{i, j} \exp \left\{i \overrightarrow{\mathbf{q}} \cdot\left(\overrightarrow{\mathbf{R}}_{\mathbf{i}}-\overrightarrow{\mathbf{R}}_{\mathbf{j}}\right)\right\}
\end{aligned}
$$

Here, $a_{d}$ refers to the structure factor of a single defect, whether a single dislocation or a dislocation pair, etc., and $c$ is a discrete finite sum that refers to the distribution of defects. We will call $c$ the "configuration factor" since it is technically not a structure factor. $\phi(\vec{x})$ is the fractional density change (dilatation) for a single defect, with the defect positioned at the origin. Of course, in a finite sample, some of the defects will be located near an external 
surface, and the boundary conditions for all the defects are thus not equivalent, but this effect can be safely ignored.

This decomposition into single-defect and configuration factors is only valid if all the defects are the same. In the general case, dislocations in a given configuration will possess different Burgers vectors and different slip planes, so they are not equivalent. In a later section, we will show how to write these factors so that they can be decomposed in the desired way; for now we work out the form for $a_{d}$, as defined above, to see how it depends on Burgers vector and slip plane, saving the generalization for later.

The first case to be treated is the single dislocation, aligned along the Z-axis. For most cases of small-angle scattering, it can be assumed that the scatterer and matrix have two distinct densities with a sharp boundary between them. For dislocations, we must consider isotropic elasticity, where only the edge component of the dislocation contributes a density change ${ }^{15}$, given by

$$
\begin{aligned}
& \phi=b_{e} \kappa \frac{\sin \theta}{u} \\
& \kappa=-\frac{1}{2 \pi} \frac{1-2 \nu}{1-\nu},
\end{aligned}
$$

where the edge component of the Burgers vector, $b_{e}$, is along the X-axis, and the polar coordinates $\theta$ and $u$ specify the position of the field point on the X-Y plane with the angle measured with respect to the $\mathrm{X}$-axis, as shown in Fig. 1. $\nu$ is Poisson's ratio.

As noted earlier, the dislocations show enough two dimensionality to make $2 \mathrm{D}$ modeling a reasonable approximation. But it is necessary to limit the distance in the "Z-direction". The reason is not that a dislocation ever ends in the crystal, but rather that a dislocation length is part of a large loop which closes on itself over some distance. More generally, the dislocation is tied into a larger structure over a distance which we can model as an effective length for the $2 \mathrm{D}$ dislocation. Thus, we integrate along the dislocation line (Z-direction) for a finite length, $-H$ to $H$,

$$
\begin{aligned}
a_{\text {single }} & =b_{e} \kappa \int_{\mathcal{R}_{0}}^{\mathcal{R}} \int_{0}^{2 \pi} \frac{\sin \theta}{u} \exp \left\{i q_{p} u \cos (\theta-\xi)\right\} u d u d \theta \int_{-H}^{H} \exp \left\{i q_{z} z\right\} d z \\
& =4 \pi b_{e} \kappa \sin \xi\left[\frac{J_{0}\left(q_{p} \mathcal{R}\right)}{q_{p}}-\frac{J_{0}\left(q_{p} \mathcal{R}_{0}\right)}{q_{p}}\right] \frac{\sin \left(q_{z} H\right)}{q_{z}} \\
& \approx 4 \pi b_{e} \kappa \frac{\sin \xi}{q_{p}} \frac{\sin \left(q_{z} H\right)}{q_{z}} .
\end{aligned}
$$

Here, $a_{\text {single }}$ is the structure factor for a single dislocation and $J_{0}$ is the zero-order Bessel function. The polar angle $\xi$ and the radial distance $q_{p}$ give the projection of the scattering vector onto the $\mathrm{X}-\mathrm{Y}$ plane. As before, the angle is measured with respect to the edge component of the Burgers vector (X-axis). In these equations, the last step is valid only when $q_{p} \mathcal{R} \gg 1$ and $q_{p} \mathcal{R}_{0} \ll 1$, where $\mathcal{R}$ is the upper cut-off for the dislocation strain field, and $\mathcal{R}_{0}$ is the lower cut-off at the effective dislocation core radius. For this derivation to be correct, it is necessary that $H \gg \mathcal{R}$. The exact expression indicates that there is no scattering by the dislocations at $q_{p}=0$, as noted by Dexter ${ }^{16}$; this must be correct, because the dislocation induces zero net volume change in the material. When the scattering vector is 
very small, it samples over a region of zero volume change, and is not scattered. These results are in the form already given by Atkinson and Hirsch ${ }^{12}$. We note here the very important point that the length factor, $\sin \left(q_{z} H /\right) / q_{z}$, is a function with a very strong and sharp peak at $q_{z}=0$ for dislocation lengths typical of deformed metals. Thus, the effective dislocation length can be directly measured in experiments that probe the angular dependence of the small-angle scattering.

The second case to be treated is a pair of dislocations displaced from one another in the Y-direction by the distance, $2 d$. See Fig. 2. The strain field of the two dislocations is expanded in a Taylor expansion up to first order. There are two regions of expansion, $u>d$ and $u<d$, but the two expansions have the same functional form, and in a straight forward extension of the above analysis, the total structure factor can be written

$$
a_{\text {dipole }} \approx \frac{8 \pi b_{e} \kappa \sin ^{2} \xi}{q_{p}^{3 / 2}} \sqrt{\frac{2}{\pi d}} \frac{\sin \left(q_{z} H\right)}{q_{z}},
$$

which is valid in the same range of $q$-space as before, and where the oscillatory part of a Bessel function is again ignored.

A comparison of Eqns. (6) and (7) shows two very important differences between scattering by single dislocations and scattering by dipoles. First, the $q$-dependences of the scattering amplitudes differ by $q_{p}^{-1 / 2}$, which results in a $q_{p}^{-1}$ difference in the scattered intensities. In the reciprocal space range where $q_{p} \approx 2 \pi / d$ and ignoring angular effects, the dipole and the single dislocation have similar scattering intensities. In USAXS experiments, however, $q_{p} \ll 2 \pi / d$, thus resulting in a much higher scattered intensity for the dipole. This is easily understood since a dipole can be regarded as a planar region of vacancies which has a net volume change at small $q_{p}$, whereas the dislocation dilatation field averages to zero. The requirement that $q_{p} \ll 2 \pi / d$ just means that the X-ray beam must sample a region larger than the defect. Second, the angular distributions of the scattering are profoundly different. The scattered intensity from dipoles goes as $\sin ^{4} \xi$ as compared with $\sin ^{2} \xi$ for single dislocations. Thus, the high relative scattering by dipoles over single dislocations will only be visible over a narrow range of orientational angles.

In addition to the elastic dilatation, which is a feature of only the edge component of the dislocation Burgers vector, dislocations possess density changes in the core, and the nonlinear part of the elastic field (even the shear component) also contributes a density change. However, as has been shown elsewhere, these contributions are negligible compared to the elastic expressions in the region of $q_{p}$ space accessible to ultra-small-angle scattering. ${ }^{12}$ The same is true of vacancies and small collections of vacancies that can form during deformation.

\section{CONFIGURATION STRUCTURE FACTORS}

\section{A. General}

The main difference between this work and that of previous authors is that we use a different factor for the dislocation configuration. Atkinson and Hirsch ${ }^{12}$ treated the dislocations as a cloud of independent scatterers. Seeger ${ }^{10}$ treated dislocation pile-ups, but these configurations are rarely observed in deformed metals. Seeger and Brand ${ }^{11}$ also considered small 
dislocation loops scattering independently, which is appropriate for an irradiated sample. In fact, it is now well understood that dislocations in deformed metals form partially-ordered structures, mostly walls, and that is the case considered here.

Dislocations in a wall can be divided into distinct populations. First, for a given slip system, there exist dislocations with both positive and negative Burgers vectors. Second, dislocations from more than one slip system may be present in a wall, depending on the deformation history and the strain geometry. Third, the dislocations may lie in different directions in the wall. Finally, some of the dislocations may be better represented as dislocation dipoles, rather than single dislocations. The analysis for this completely general case is quite complicated, and simplifying assumptions must be made to build a useful physical picture. In particular, to obtain a usable solution, it is necessary to achieve a decomposition into a number of factors, and the first goal in this section is to show under what conditions this can be achieved for the single defect and configuration factors.

Factorization cannot be achieved if both dipoles and dislocations coexist in a wall, because the single defect functional dependence on $q_{p}$ is different for the two cases. But if only one class of defect, either single dislocations or dipoles, is dominant, then the functional form for this scattering can be separated from all other configurational considerations. As shall be seen later, however, there is a range in reciprocal space where the contributions of single dislocations and dipoles can be separated and direct experimental measurements of the relative numbers of these defects may be possible.

The problem can be simplified further by focusing on single-crystal fcc metals, with $\{111\}\langle 110\rangle$ slip systems. In the early stages of deformation, i.e. Stage II, walls often develop paraliel to the $\{111\}$ slip planes-the "carpets" referred to in the Introduction. The dislocations are generally parallel to one another, and may take the form of partiallyordered Taylor lattices ${ }^{2}$. In later stages of deformation, walls are formed roughly normal to the primary slip planes, presumably the end result of cross slip and climb from the initial slip planes, or the interaction between two slip planes operating simultaneously. For example, two separate slip planes will intersect in $\langle 110\rangle$ type directions. Dislocation walls created by such an interaction contain Burgers vectors from two different slip planes, but the dislocations will all be parallel to the intersection line of the two slip planes. Thus, for either the carpets or the stage III walls, it is reasonable to assume that the dislocations in a given wall all lie essentially parallel to one another, even though they will have both Burgers vector sign, and may even have more than one Burgers vector direction on more than one slip plane. If the defects all lie parallel to one another, it is possible to factor out the line direction part of the scattering function, and a further factorization has been achieved.

We start by rewriting the structure factor for an individual defect, $i$, as a product,

$$
a_{i}=\beta_{i} \mathcal{A}_{i} f_{i}(\xi) .
$$

In this equation, since the defects can belong to more than one slip system, they may have a variety of Burgers vector edge components, $\beta_{i}= \pm\left(b_{e}\right)_{i}$, and angular factors, $f_{i}(\xi)$. As shown in Eqns. $(6,7), f$ and $\mathcal{A}$ have different functional forms for single dislocations and for dipoles. However, $\mathcal{A}$ does not depend on the sign of the Burgers vector or the particular slip plane. With the above notation, we include the Burgers vector and angular factors, $\beta_{i}$ and $f_{i}(\xi)$, in the configuration factors, and write $\mathcal{W}$ for the new configuration factor, 


$$
\mathcal{W}=\sum_{i, j} \beta_{i} \beta_{j} f_{i}(\xi) f_{j}(\xi) \exp \left\{i \overrightarrow{\mathbf{q}} \cdot\left(\overrightarrow{\mathbf{R}}_{\mathbf{i}}-\overrightarrow{\mathbf{R}}_{\mathbf{j}}\right)\right\}
$$

where, from Eqns. $(6,7)$,

$$
\begin{aligned}
\mathcal{I} & =\mathcal{A}^{2} \mathcal{W} \\
\mathcal{A}_{\text {single }} & \approx \frac{4 \pi \kappa}{q_{p}}\left(\frac{\sin \left(q_{z} H\right)}{q_{z}}\right) \\
\mathcal{A}_{\text {dipole }} & \approx \frac{8 \pi \kappa}{q_{p}^{3 / 2}} \sqrt{\frac{2}{\pi d}}\left(\frac{\sin \left(q_{z} H\right)}{q_{z}}\right) .
\end{aligned}
$$

Although this separation of $\mathcal{A}$ and $\mathcal{W}$ is only rigorously valid for materials that satisfy the earlier assumption of isotropic elasticity, the general results of this paper should be substantially the same for anisotropic crystals.

These equations are valid only if a particular defect type (dipole or single dislocation) is dominant in a given wall. If both dipoles and single dislocations exist in significant numbers in the same wall, then the scattered intensity cannot be factored in this way, and interference between the two types of defects will be present. A second proviso is that Eqn. (9) applies to a single wall. Scattered intensity from more than one wall is assumed to be incoherent, and is simply a sum over the different walls. For $q<0.0002 \AA^{-1}$, at large strains in $\mathrm{Cu}$ or $\mathrm{Al}$ solid solution alloys, complete cells will scatter; but this type of scattering is not yet experimentally accessible, and is not the subject of this paper.

Equations (9) and (10) describe the scattering from a specific configuration of dislocations or dipoles in a particular wall. For the averaged scattering over a collection of similar walls, the averaged intensity becomes

$$
\langle\mathcal{I}\rangle=\mathcal{A}^{2}\langle\mathcal{W}\rangle
$$

Now it is clear why the defect structure factor, $a_{d}$, introduced in Eqn. (3), is not the appropriate expression when averages over probability distributions must be taken; only $\mathcal{A}$ is invariant for all defects of a given type (i.e. single dislocation or dipole) in a distribution of defects.

The analysis of $\langle\mathcal{W}\rangle$ forms the content of the remainder of this subsection. First, a joint pair distribution function is defined for the double sum over pairs in Eqn. (9). Let $\wp\left(\overrightarrow{\mathbf{R}}_{i}, \overrightarrow{\mathbf{R}}_{j}\right)$ be defined as the joint probability for finding one dislocation, labeled $i$ at $\overrightarrow{\mathbf{R}}_{i}$ and a second dislocation labeled $j$ at $\overrightarrow{\mathbf{R}}_{j}$. Then, following (9), $\langle\mathcal{W}\rangle$ is written

$$
\begin{aligned}
& \langle\mathcal{W}\rangle=\sum_{i, j} \beta_{i} \beta_{j} f_{i}(\xi) f_{j}(\xi) \iint \wp\left(\overrightarrow{\mathbf{R}}_{i}, \overrightarrow{\mathbf{R}}_{j}\right) \exp \left\{i \overrightarrow{\mathbf{q}} \cdot\left(\overrightarrow{\mathbf{R}}_{i}-\overrightarrow{\mathbf{R}}_{j}\right)\right\} d \overrightarrow{\mathbf{R}}_{i} d \overrightarrow{\mathbf{R}}_{j} \\
& \iint \wp\left(\overrightarrow{\mathbf{R}}_{i}, \overrightarrow{\mathbf{R}}_{j}\right) d \overrightarrow{\mathbf{R}}_{i} d \overrightarrow{\mathbf{R}}_{j}=1
\end{aligned}
$$

where the second equation gives the normalization used for the joint pair distribution function. In the double sum over the dislocation pairs, the diagonal terms for $i=j$ refer to the same dislocation, and represent the self scattering of the separate dislocations. For these self scattering terms, of course, $\wp=\delta\left(\overrightarrow{\mathbf{R}}_{i}, \overrightarrow{\mathbf{R}}_{i}\right)$. For all pairs, however, where the dislocations 
making up the pair are different dislocations, the joint pair distribution function will be assumed to be a smooth function. Thus, we can write

$$
\begin{aligned}
\langle\mathcal{W}\rangle & =\sum_{i} \beta_{i}^{2} f_{i}^{2}(\xi) \\
& +\sum_{i \neq j} \beta_{i} \beta_{j} f_{i}(\xi) f_{j}(\xi) \iint \wp\left(\overrightarrow{\mathbf{R}}_{i}, \overrightarrow{\mathbf{R}}_{j}\right) \exp \left\{i \overrightarrow{\mathbf{q}} \cdot\left(\overrightarrow{\mathbf{R}}_{i}-\overrightarrow{\mathbf{R}}_{j}\right)\right\} d \overrightarrow{\mathbf{R}}_{i} d \overrightarrow{\mathbf{R}}_{j} .
\end{aligned}
$$

Now remember that the dislocations consist of several populations with different Burgers vectors, slip planes, etc., and that within each type, e.g. a given Burgers vector, etc., the dislocations are equivalent and have equal $\wp\left(\overrightarrow{\mathbf{R}}_{i}, \overrightarrow{\mathbf{R}}_{j}\right)$. Thus the sums over dislocations of a given type can be replaced by $N_{m}$, etc., where $N_{m}$ refers to the number of dislocations of type $m$. Accordingly, the component parts of $\langle\mathcal{W}\rangle=\left\langle\mathcal{W}_{m m}\right\rangle+\left\langle\mathcal{W}_{m n}\right\rangle$, can be written,

$$
\begin{aligned}
\left\langle\mathcal{W}_{m m}\right\rangle & =\sum_{m} N_{m} \beta_{m}^{2} f_{m}^{2}(\xi) \\
& +\sum_{m} N_{m}\left(N_{m}-1\right) \beta_{m}^{2} f_{m}^{2}(\xi) \iint \mathcal{P}\left(\overrightarrow{\mathbf{R}}_{m}, \overrightarrow{\mathbf{R}}_{m}^{\prime}\right) \exp \left\{i \overrightarrow{\mathbf{q}} \cdot\left(\overrightarrow{\mathbf{R}}_{m}-\overrightarrow{\mathbf{R}}_{m}^{\prime}\right)\right\} d \overrightarrow{\mathbf{R}}_{m} d \overrightarrow{\mathbf{R}}_{m}^{\prime}, \\
\left\langle\mathcal{W}_{m n}\right\rangle & =\sum_{m \neq n} N_{m} N_{n} \beta_{m} \beta_{n} f_{m}(\xi) f_{n}(\xi) \\
& \times \iint \mathcal{P}\left(\overrightarrow{\mathbf{R}}_{m}, \overrightarrow{\mathbf{R}}_{n}^{\prime}\right) \exp \left\{i \overrightarrow{\mathbf{q}} \cdot\left(\overrightarrow{\mathbf{R}}_{m}-\overrightarrow{\mathbf{R}}_{n}^{\prime}\right)\right\} d \overrightarrow{\mathbf{R}}_{m} d \overrightarrow{\mathbf{R}}_{n}^{\prime},
\end{aligned}
$$

where the first equation refers to dislocations belonging to the same type, and the second to dislocations of different types. The factor, $N_{m}\left(N_{m}-1\right)$ in this equation arises because there are $N_{m}\left(N_{m}-1\right)$ terms in the off-diagonal double sum for each dislocation type in the second term of (13). The new joint pair distribution function, $\mathcal{P}\left(\overrightarrow{\mathbf{R}}_{m}, \overrightarrow{\mathbf{R}}_{n}^{\prime}\right)$ is now the joint probability of finding one dislocation of type $m$ at position $\overrightarrow{\mathbf{R}}_{m}$ and a second (different) dislocation of type $n$ at $\overrightarrow{\mathbf{R}}_{n}^{\prime}$. (Of course, the dislocations may be of the same type, $m=n$ or of different types, $m \neq n$.) It is very important to note that this joint pair distribution function is not the standard pair correlation function. These equations can be combined into the final equations,

$$
\begin{aligned}
& \langle\mathcal{W}\rangle=\sum_{m} N_{m} \beta_{m}^{2} f_{m}^{2}(\xi)\left[1-\iint \mathcal{P}\left(\overrightarrow{\mathbf{R}}_{m}, \overrightarrow{\mathbf{R}}_{m}^{\prime}\right) \exp \left\{i \overrightarrow{\mathbf{q}} \cdot\left(\overrightarrow{\mathbf{R}}_{m}-\overrightarrow{\mathbf{R}}_{m}^{\prime}\right)\right\} d \overrightarrow{\mathbf{R}}_{m} d \overrightarrow{\mathbf{R}}_{m}^{\prime}\right] \\
& +\sum_{m, n} N_{m} N_{n} \beta_{m} \beta_{n} f_{m}(\xi) f_{n}(\xi) \iint \mathcal{P}\left(\overrightarrow{\mathbf{R}}_{m}, \overrightarrow{\mathbf{R}}_{n}^{\prime}\right) \exp \left\{i \overrightarrow{\mathbf{q}} \cdot\left(\overrightarrow{\mathbf{R}}_{m}-\overrightarrow{\mathbf{R}}_{n}^{\prime}\right)\right\} d \overrightarrow{\mathbf{R}}_{m} d \overrightarrow{\mathbf{R}}_{n}^{\prime} \\
& \iint \mathcal{P}\left(\overrightarrow{\mathbf{R}}_{m}, \overrightarrow{\mathbf{R}}_{n}^{\prime}\right) d \overrightarrow{\mathbf{R}}_{m} d \overrightarrow{\mathbf{R}}_{n}^{\prime}=1
\end{aligned}
$$

where the double sum is taken over all $(m, n)$. The last equation sets the normalization for the joint pair distribution functions.

Eqn. (15) has a very important form. The first term, the one with unity in the brackets, is the self scattering term, which is independent of the distribution function because a given dislocation is fully correlated with itself. It is also independent of the scattering vector, $q$, and is proportional to the number of scatterers, $N_{m}$. This first term will be seen to have special importance in the following for the case where the net Burgers vector in a wall is near zero. 
Finally, we note that although it is physically impossible for two different dislocations to occupy the same position, this does not mean that we must require $\mathcal{P}\left(\overrightarrow{\mathbf{R}}_{m}, \overrightarrow{\mathbf{R}}_{n}^{\prime}\right)=0$ for $\overrightarrow{\mathbf{R}}_{m}=\overrightarrow{\mathbf{R}}_{n}^{\prime}$. Although physically incorrect, we will show in a later section that the resulting error is insignificant. This allows us to use much simpler distribution functions in the analysis.

\section{B. Walls of Zero Thickness}

Because the $\vec{R}$ dependence of the exponential in Eqn. (15) is only a function of the difference between the dislocation positions, it is natural to introduce a change of variables,

$$
\begin{aligned}
& R_{m}+R_{n}^{\prime}=2 r_{m n}^{\prime} \\
& R_{m}-R_{n}^{\prime}=r_{m n} .
\end{aligned}
$$

Here, it is assumed that the wall has zero thickness; in the next subsection, the results are generalized to finite thickness. Thus, the position vectors in Eqn. (15) become scalars, and the product in the exponential will be chosen such that $q_{w}$ lies in the wall, normal to the dislocation lines. Because the integration is finite, one must integrate over the appropriate limits. For a wall which extends from $R=0$ to $R=+L$, the initial and transformed integration spaces are shown in Fig. 3. Note that the Jacobian for the transformation is 1 , and $d R d R^{\prime}=d r d r^{\prime}$. It is reasonable to assume that the probability distribution for the dislocations depends only on their relative positions, $r$, and not on their average positions, $r^{\prime}$. With this assumption, the integral over $r^{\prime}$ is

$$
\begin{aligned}
& \iint \mathcal{P}\left(R_{m}, R_{n}^{\prime}\right) \cos \left\{q_{w}\left(R_{m}-R_{n}^{\prime}\right)\right\} d R_{m} d R_{n}^{\prime} \\
& =\iint \mathcal{P}\left(r_{m n}, r_{m n}^{\prime}\right) \cos \left\{q_{w} r_{m n}\right\} d r_{m n} d r_{m n}^{\prime} \\
& =\int \mathcal{P}\left(r_{m n}\right) \cos \left\{q_{w} r_{m n}\right\} d r_{m n} \int d r_{m n}^{\prime} .
\end{aligned}
$$

The two integrals in the last line are to be integrated over the rotated rhombus in Fig. 3. Because of the symmetry about the $r_{m n}^{\prime}$ axis, the integral only needs to be carried out over the upper triangle of the rhombus, for $r_{m n}>0$. Thus,

$$
\begin{aligned}
& \int \mathcal{P}\left(r_{m n}\right) \cos \left\{q_{w} r_{m n}\right\} d r_{m n} \int d r_{m n}^{\prime} \\
& =2 \int_{0}^{L} \mathcal{P}\left(r_{m n}\right) \cos \left\{q_{w} r_{m n}\right\} d r_{m n} \int_{r_{m n} / 2}^{L-r_{m n} / 2} d r_{m n}^{\prime} \\
& =2 \int_{0}^{L}\left(L-r_{m n}\right) \mathcal{P}\left(r_{m n}\right) \cos \left\{q_{w} r_{m n}\right\} d r_{m n} .
\end{aligned}
$$

The normalization of $\mathcal{P}\left(r_{m n}\right)$ follows from that for the total distribution function,

$$
\begin{aligned}
1 & =\iint \mathcal{P}\left(R_{m}, R_{n}^{\prime}\right) d R_{m} d R_{n}^{\prime}=\iint \mathcal{P}\left(r_{m n}, r_{m n}^{\prime}\right) d r_{m n} d r_{m n}^{\prime} \\
& =2 \int_{0}^{L} \mathcal{P}\left(r_{m n}\right)\left(L-r_{m n}\right) d r_{m n} .
\end{aligned}
$$


When all dislocations are completely random, it follows that $\mathcal{P}_{\text {random }}=1 / L^{2}$.

The function, $\mathcal{P}\left(r_{m n}\right)$, requires special attention. From its definition, $\mathcal{P}\left(R_{m}, R_{n}^{\prime}\right)=$ $\mathcal{P}\left(r_{m n}, r_{m n}^{\prime}\right)=\mathcal{P}\left(r_{m n}\right)$, it remains a joint pair distribution function, even though it depends only on the single variable, $r_{m n}$, the pair separation distance. Thus, $\mathcal{P}\left(r_{m n}\right)$ should not be interpreted as the probability of finding a dislocation pair with separation $r_{m n}$.

Using Eqns. (17) and (18) in Eqn. (15), the configuration factor, $\langle\mathcal{W}\rangle$, becomes

$$
\begin{aligned}
\langle\mathcal{W}\rangle & =\sum_{m} N_{m} \beta_{m}^{2} f_{m}^{2}(\xi)\left(1-\left\langle\mathcal{W}_{m m}\right\rangle\right)+\sum_{m, n} N_{m} N_{n} \beta_{m} \beta_{n} f_{m}(\xi) f_{n}(\xi)\left\langle\mathcal{W}_{m n}\right\rangle \\
\left\langle\mathcal{W}_{m n}\right\rangle & =2 \int_{0}^{L}\left(L-r_{m n}\right) \mathcal{P}\left(r_{m n}\right) \cos \left(q_{w} r_{m n}\right) d r_{m n} .
\end{aligned}
$$

This is a very general equation, depending only upon the joint pair distribution functions for the pairs, $(m n)$, within the populations. Note that, in general, correlations exist between pairs of plus and minus Burgers vectors, and between dislocation pairs on different slip planes. Recall also that it is assumed that only the distances between the dislocations of a pair are correlated. The midpoint of the pair is randomly distributed over the wall, and is uncorrelated with other pair midpoints.

The part of the sum over populations which relates to Burgers vector sign can be further manipulated if the excess sign (proportional to the net Burgers vector), $B_{s}$, and the total number of dislocations, $N_{s}$ for each slip system, $s$, are introduced,

$$
\begin{aligned}
& B_{s}=N_{s}^{+}-N_{s}^{-} \\
& N_{s}=N_{s}^{+}+N_{s}^{-} .
\end{aligned}
$$

Then the sum over the positive and negative dislocations for a given slip plane group can be carried out explicitly to give

$$
\begin{aligned}
\langle\mathcal{W}\rangle & =\sum_{s} b_{e}^{2}(s) f_{s}^{2}(\xi)\left(N_{s}-N_{s}^{+}\left\langle\mathcal{W}_{s s}^{++}\right\rangle-N_{s}^{-}\left\langle\mathcal{W}_{s s}^{--}\right\rangle\right) \\
& +\sum_{s, s^{\prime}} \frac{b_{e}(s) b_{e}\left(s^{\prime}\right) f_{s}(\xi) f_{s^{\prime}}(\xi)}{4}\left[\left(N_{s} N_{s^{\prime}}+B_{s} B_{s^{\prime}}\right)\left(\left\langle\mathcal{W}_{s s^{\prime}}^{++}\right\rangle+\left\langle\mathcal{W}_{s s^{\prime}}^{--}\right\rangle\right)\right. \\
& \left.+\left(N_{s} B_{s^{\prime}}+N_{s^{\prime}} B_{s}\right)\left(\left\langle\mathcal{W}_{s s^{\prime}}^{++}\right\rangle-\left\langle\mathcal{W}_{s s^{\prime}}^{--}\right\rangle\right)-2\left(N_{s} N_{s^{\prime}}-B_{s} B_{s^{\prime}}\right)\left\langle\mathcal{W}^{+-}\right\rangle\right] \\
\left\langle\mathcal{W}_{s s^{\prime}}^{++}\right\rangle & =2 \int_{0}^{L}\left(L-r_{s s^{\prime}}\right) \mathcal{P}^{++}\left(r_{s s^{\prime}}\right) \cos \left(q_{w} r_{s s^{\prime}}\right) d r_{s s^{\prime}}, \text { etc. },
\end{aligned}
$$

where the configuration factors $\left\langle\mathcal{W}_{s s^{\prime}}^{++}\right\rangle$refer to both dislocations positive and $\left\langle\mathcal{W}_{s s^{\prime}}^{+-}\right\rangle$refer to unlike sign Burgers vectors. It is assumed that the configuration factors satisfy the relations

$$
\left\langle\mathcal{W}_{s s^{\prime}}^{+-}\right\rangle=\left\langle\mathcal{W}_{s s^{\prime}}^{-+}\right\rangle
$$

Again, these equations are quite general, and describe different distributions of like and unlike signs within a given slip plane set, and of course also allow for different distributions between slip planes. The equations are complicated, but that is a reflection of the 
complicated nature of the dislocation ordering problem. For example, when one sign of Burgers vector (say -) is in the minority, then ordering will be dominated by the majority population, and the minority will tend to be disordered (relative to itself), with possibly strong correlations between unlike dislocations. Thus, $\left\langle\mathcal{W}_{s s}^{++}\right\rangle$and $\left\langle\mathcal{W}_{s s}^{+-}\right\rangle$may be strongly correlated, while $\left\langle\mathcal{W}_{s s}^{--}\right\rangle$may be nearly random since the average negative dislocation separation greatly exceeds the relevant correlation distances. On the other hand, for $B=0$, all distribution functions are likely to be strongly correlated. Since this $B=0$ case is of great interest for deformed metals, we return to it at the end of §IIID.

When the populations are fully disordered, then a good approximation will be

$$
\left\langle\mathcal{W}_{s s^{\prime}}^{0}\right\rangle=\left\langle\mathcal{W}_{s s^{\prime}}^{++}\right\rangle=\left\langle\mathcal{W}_{s s^{\prime}}^{--}\right\rangle=\left\langle\mathcal{W}_{s s^{\prime}}^{+-}\right\rangle
$$

and one can write the very simple result

$$
\langle\mathcal{W}\rangle=\sum_{s} b_{e}^{2}(s) N_{s} f_{s}^{2}(\xi)\left(1-\left\langle\mathcal{W}_{s s}^{0}\right\rangle\right)+\sum_{s, s^{\prime}} b_{e}(s) b_{e}\left(s^{\prime}\right) f_{s}(\xi) f_{s^{\prime}}(\xi) B_{s} B_{s^{\prime}}\left\langle\mathcal{W}_{s s^{\prime}}^{0}\right\rangle
$$

Thus, if there are no special correlations between like and unlike sign dislocations, the walls are most transparent when the net number of positive and negative dislocations are equal and $B_{s}=B_{s^{\prime}}=0$, with only the term linear in the $N$ contributing. This linear term can be simplified further by solving explicitly for $\left\langle\mathcal{W}_{s s}^{0}\right\rangle$. For completely uncorrelated dislocations within a single slip plane,

$$
\begin{aligned}
\left\langle\mathcal{W}_{s s}^{0}\right\rangle & =\frac{\sin ^{2}\left(q_{w} L / 2\right)}{q_{w}^{2} L^{2} / 4} \\
\langle\mathcal{W}\rangle & =b_{e}^{2} N f^{2}(\xi)\left(1-\frac{\sin ^{2}\left(q_{w} L / 2\right)}{q_{w}^{2} L^{2} / 4}\right) .
\end{aligned}
$$

This equation has some very interesting properties. First, as $q_{w} \rightarrow 0$, the scattering goes to zero quadratically. Second, as the scattering vector increases such that $q_{w} L \gg 1$, the scattering goes to the self scattering limit, proportional to the total number of dislocations, $N$. Experimentally, it will usually be the case that $q_{w} L \gg 1$, so the wall function for $B=0$ is a constant, independent of $q_{w}$. The fact that the wall function goes to zero at $q_{w}=0$ in this case is simply due to the fact that we are then sampling over a region with no net volume change. The reader should not confuse this behavior of $\langle\mathcal{W}\rangle$ as $q_{w} \rightarrow 0$ with the near zero behavior of the single dislocation structure factor of $§ I I$. In particular, $q_{w}$ in Eqn. (26) and $q_{p}$ in Eqn. (6) are generally not equal and the widths of the two holes at the origin are also different.

During the early stages of uniaxial deformation, the walls contain nearly equal numbers of positive and negative dislocations. But one does not expect the configuration factors to be exactly equal for the $(++)$ and $(+-)$ cases. We return later to the case where the dislocations are partially ordered.

Now compare this result for $B=0$ with the case of a totally disordered wall containing only positive dislocations from a single slip-system $(B=N)$. Equation (25) can then be integrated immediately, with $\mathcal{P}(r)=1 / L^{2}$ to give,

$$
\langle\mathcal{W}\rangle=b_{e}^{2} N f^{2}(\xi)\left(1-\frac{\sin ^{2}\left(q_{w} L / 2\right)}{q_{w}^{2} L^{2} / 4}\right)+b_{e}^{2} N^{2} f^{2}(\xi) \frac{\sin ^{2}\left(q_{w} L / 2\right)}{q_{w}^{2} L^{2} / 4}
$$


The second term only dominates if $q_{w} \ll \sqrt{N / L^{2}}$. For realistic experimental conditions, this requirement can only be satisfied if $\overrightarrow{\mathbf{q}}_{w}$ is nearly perpendicular to $\overrightarrow{\mathbf{q}}$. The second term is the standard optics diffraction function for a single slit the size of the wall.

Thus, when the dislocations are totally random, and $q_{w} \gg \sqrt{N / L^{2}}$, the scattering is the same for both $B=0$ and for $B=N$. As $q_{w} \rightarrow 0$, the scattering will be proportional to the square of the uncompensated Burgers vector.

In using these equations, one should be guided by the apparent fact that in a deforming metal, any given region will tend to be in single slip, with a preponderance of dislocations from a single slip plane. ${ }^{8}$ The conjugate systems are probably important as a means to form "hard spots" which tie the configuration down to the underlying lattice, but usually in any given local region, these dislocations are only a small minority in the total dislocation population. Thus, for scattering purposes, it may be appropriate to use the equations as if only a single slip system is operating for a given wall, and average over the different slip systems only when the intensities of individual walls are averaged.

\section{Walls of Non-zero Thickness}

The walls of dislocations formed during deformation are probably diffuse and have a nonzero thickness. In general, the mechanisms responsible for wall formation are different in the length and thickness directions. For example, if a wall is perpendicular to the slip plane of the component dislocations, then the formation mechanism in the length direction would include dislocation climb and source distributions whereas the thickness formation mechanism would include elastic interactions and glide. Therefore, it is reasonable to assume that the dislocation distributions in the thickness direction and in the length direction are independent, so that $\mathcal{P}\left(w, t ; w^{\prime}, t^{\prime}\right)=\mathcal{P}\left(w, w^{\prime}\right) \mathcal{P}\left(t, t^{\prime}\right)$, where $t$ and $w$ are the coordinates of a dislocation in the thickness and length directions, respectively. Then $\langle\mathcal{W}\rangle$ can be written

$$
\begin{aligned}
\langle\mathcal{W}\rangle & =\sum_{m} N_{m} \beta_{m}^{2} f_{m}^{2}(\xi)\left(1-2 \iint \mathcal{P}\left(w_{m}, w_{m}^{\prime}\right) \cos \left(q_{t} x_{m m}\right) d w_{m} d w_{m}^{\prime}\right. \\
& \left.\times \int_{0}^{L}\left(L-y_{m m}\right) \mathcal{P}\left(y_{m m}\right) \cos \left(q_{w} y_{m m}\right) d y_{m m}\right) \\
& +2 \sum_{m, n} N_{m} N_{n} \beta_{m} \beta_{n} f_{m}(\xi) f_{n}(\xi) \iint \mathcal{P}\left(w_{m}, w_{n}^{\prime}\right) \cos \left(q_{t} x_{m n}\right) d w_{m} d w_{n}^{\prime} \\
& \times \int_{0}^{L}\left(L-y_{m n}\right) \mathcal{P}\left(y_{m n}\right) \cos \left(q_{w} y_{m n}\right) d y_{m n}
\end{aligned}
$$

where

$$
\begin{aligned}
w_{m}-w_{n}^{\prime} & =x_{m n} \\
w_{m}+w_{n}^{\prime} & =2 x_{m n}^{\prime} \\
t_{m}-t_{n}^{\prime} & =y_{m n} \\
t_{m}+t_{n}^{\prime} & =2 y_{m n}^{\prime}
\end{aligned}
$$

are the equations for transforming the coordinates into the "rotated" coordinates suitable for the integrations, just as before. To simplify the analysis, we split the total wall factor into factors for the thickness, $\left\langle\mathcal{W}_{m n}^{t}\right\rangle$, and length, $\left\langle\mathcal{W}_{m n}^{w}\right\rangle$, components, 


$$
\begin{aligned}
\langle\mathcal{W}\rangle & =\sum_{m} N_{m} \beta_{m}^{2} f_{m}^{2}(\xi)\left(1-\left\langle\mathcal{W}_{m m}^{t}\right\rangle\left\langle\mathcal{W}_{m m}^{w}\right\rangle\right) \\
& +\sum_{m, n} N_{m} N_{m} \beta_{m} \beta_{n} f_{m}(\xi) f_{n}(\xi)\left\langle\mathcal{W}_{m n}^{t}\right\rangle\left\langle\mathcal{W}_{m n}^{w}\right\rangle \\
\left\langle\mathcal{W}_{m n}^{t}\right\rangle & =\iint \mathcal{P}\left(w_{m}, w_{n}^{\prime}\right) \cos \left(q_{t} x_{m n}\right) d w_{m} d w_{n}^{\prime} \\
\left\langle\mathcal{W}_{m n}^{w}\right\rangle & =2 \int_{0}^{L}\left(L-y_{m n}\right) \mathcal{P}\left(y_{m n}\right) \cos \left(q_{w} y_{m n}\right) d y_{m n}
\end{aligned}
$$

We will explore two simple cases for $\left\langle\mathcal{W}_{m n}^{t}\right\rangle$. In the first case, the dislocations are randomly distributed over a fixed width. Then $\mathcal{P}\left(w_{m}, w_{n}^{\prime}\right)=1 / l_{t}^{2}$, for $0<w_{m}, w_{n}^{\prime}<l_{t}$, where $l_{t}$ is the wall thickness. Such a wall has sharp boundaries at $w=0, l_{t}$. Then, when the coordinate transformation is invoked for $w_{m}, w_{n}^{\prime}$, as before, and the integrations are carried out over $x_{m n}$ and $x_{m n}^{\prime}$,

$$
\left\langle\mathcal{W}_{m n}^{t}\right\rangle=\frac{\sin ^{2}\left(q_{t} l_{t} / 2\right)}{q_{t}^{2} l_{t}^{2} / 4}
$$

This thickness factor is the familiar single-slit diffraction function that was found in Eqn. (26), and has a power law fall off at high $q_{t}$ of $q_{t}^{-2}$. If the wall is sufficiently thin such that $q_{t} l_{t} \ll 1$, then $\left\langle\mathcal{W}_{m n}^{t}\right\rangle=1$, and the result reverts to that of the previous section.

A second simple case for a wall of finite thickness can be obtained by assuming that the distribution function in the width direction is Gaussian. Thus,

$$
\mathcal{P}\left(w_{m}\right)=\frac{1}{l_{t} \sqrt{\pi}} e^{-\left(w_{m} / l_{t}\right)^{2}},
$$

where $l_{t}$ is the width of the distribution, and the total joint pair distribution function in the thickness direction is $\mathcal{P}\left(w_{m}\right) \mathcal{P}\left(w_{n}^{\prime}\right)$. Taking the same transformation of coordinates $w_{m}, w_{n}^{\prime}$ into $x_{m n}, x_{m n}^{\prime}$ as given in Eqn. (29), the joint pair distribution in $x_{m n}, x_{m n}^{\prime}$ space is

$$
\mathcal{P}\left(x_{m n}, x_{m n}^{\prime}\right)=\mathcal{P}\left(w_{m}\right) \mathcal{P}\left(w_{n}^{\prime}\right)=\frac{1}{\pi l_{t}^{2}} \exp \left(-\frac{1}{l_{t}^{2}}\left(x_{m n}^{\prime 2}+x_{m n}^{2} / 2\right)\right),
$$

so that the width part of the distribution function is again a product, and $\left\langle\mathcal{W}_{m n}^{t}\right\rangle$ can be written

$$
\begin{aligned}
\left\langle\mathcal{W}_{m n}^{t}\right\rangle & =\int_{-\infty}^{\infty} \int_{-\infty}^{\infty} \mathcal{P}\left(x_{m n}, x_{m n}^{\prime}\right) \cos \left(q_{t} x_{m n}\right) d x_{m n} d x_{m n}^{\prime} \\
& =\frac{2}{l_{t} \sqrt{\pi}} \int_{0}^{\infty} \exp \left(-\frac{x_{m n}^{2}}{4 l_{t}^{2}}\right) \cos \left(q_{t} x_{m n}\right) d x_{m n} \\
& =\sqrt{2} \exp \left(-\frac{1}{2} q_{t}^{2} l_{t}^{2}\right) .
\end{aligned}
$$

This equation is similar to the sharp (thick) wall case, except that $\left\langle\mathcal{W}_{m n}^{t}\right\rangle$ is a Gaussian instead of a single-slit diffraction function. Both distributions have widths of $l_{t}$, but the scattering by the sharp wall has a $1 / q_{t}^{2}$ power law fall off at large $q_{t}$, while the scattering by the diffuse wall decreases more rapidly. 
It is important to note an essential constraint in the analysis of $\left\langle\mathcal{W}_{m n}^{t}\right\rangle$. In the earlier analysis of $\left\langle\mathcal{W}_{m n}^{w}\right\rangle$, we made the assumption that the distribution function, $\mathcal{P}\left(y_{m n}, y_{m n}^{\prime}\right)$, can be factored, $\mathcal{P}\left(y_{m n}, y_{m n}^{\prime}\right)=\mathcal{P}\left(y_{m n}\right) \mathcal{P}\left(y_{m n}^{\prime}\right)$. This means that there is no correlation between the distribution of pair distances and pair centers. But when a pair is near the end of the wall, this assumption must break down, because a pair must sense the end of the wall, and must not overlap it. That is, the joint pair distribution function, $\mathcal{P}\left(y_{m n}\right)$ must also depend on the position of the midpoint, $y_{m n}^{\prime}$. This error is not important if, as expected, the length of the wall is very large compared to the dislocation correlation length. In this case, only a very small percentage of the dislocations fail to follow the factored distribution function. But for the wall width analysis, the wall width may not be very much larger than the corresponding dislocation correlation length. In this case, it is not valid to split $\mathcal{P}\left(x_{m n}, x_{m n}^{\prime}\right)$ into independent factors. Thus, except for special cases like those explored above, one cannot go further than the first equation of the width analysis, Eqn. (28), in a rigorous general treatment.

Another approach for the treatment ${ }^{19}$ of diffuse boundaries in small-angle scattering has proven very useful for the interpretation of scattering by block co-polymers with diffuse interfaces. We now adapt that approach to the case of scattering by a dislocation wall.

If the dislocation pair distribution function is uncorrelated, it follows that $\mathcal{P}\left(w_{m}, w_{n}^{\prime}\right)=$ $\mathcal{P}\left(w_{m}\right) \mathcal{P}\left(w_{n}^{\prime}\right)$, and

$$
\begin{aligned}
\left\langle\mathcal{W}_{m n}^{t}\right\rangle & =\iint \mathcal{P}\left(w_{m}, w_{n}^{\prime}\right) \cos \left(q_{t} x_{m n}\right) d w_{m} d w_{n}^{\prime} \\
& =\int\left[\int \mathcal{P}\left(x_{m n}+w_{n}^{\prime}\right) \mathcal{P}\left(w_{n}^{\prime}\right) d w_{n}^{\prime}\right] \cos \left(q_{t} x_{m n}\right) d x_{m n} \\
& =\mathcal{F}(\mathcal{P} \otimes \mathcal{P}) .
\end{aligned}
$$

In this equation, $\mathcal{F}$ is the (1D) Fourier transform, and $\otimes$ denotes the Fourier convolution of two functions. Following Ruland ${ }^{20}$ (see also Koberstein, et al. ${ }^{19}$ ), a wall with a diffuse boundary is introduced as the convolution between a probability function, $\mathcal{P}_{0}\left(w_{m}\right)$, representing a sharp wall with width $l_{t}$, and a smoothing function, $S\left(w_{m}\right)$. The smoothing function goes uniformly to zero for large $w_{m}$ and has a diffuse width, $l_{t d}$. The probability function for the non-sharp wall thus has the form

$$
\mathcal{P}\left(w_{m}\right)=S\left(w_{m}\right) \otimes \mathcal{P}_{0}\left(w_{m}\right) .
$$

Using the Fourier convolution theorem, we obtain

$$
\left\langle\mathcal{W}_{m n}^{t}\right\rangle=\mathcal{F}\left(\mathcal{P}_{0} \otimes S \otimes \mathcal{P}_{0} \otimes S\right)=\left\langle\mathcal{W}_{m n}^{t 0}\right\rangle[\mathcal{F}(S)]^{2} \approx \frac{4}{q_{t}^{2} l_{t}^{2}}[\mathcal{F}(S)]^{2},
$$

where $\left\langle\mathcal{W}_{m n}^{t 0}\right\rangle$ is the sharp wall factor. The last expression is valid for large $q_{t} l_{t}$, and $[\mathcal{F}(S)]^{2}$ will be a function of $q_{t}$ which sharpens the $q^{-2}$ law for large $q_{t}$.

Given a model for the smoothing function, $S$, the observed deviation from $q^{-2}$ scattering can be used to obtain an estimate for $l_{t d}$. This issue has been addressed extensively, most notably again by Ruland ${ }^{20}$ and Koberstein, et al. ${ }^{19}$. The most common approach is to use the sigmoidal-gradient model in which the smoothing function is assumed to be Gaussian. The resulting thickness part of the wall factor is then given by 


$$
\left\langle\mathcal{W}_{m n}^{t}\right\rangle \approx \frac{4}{q_{t}^{2} l_{t}^{2}}\left(1-\sigma^{2} q_{t}^{2}\right)
$$

where $\sigma$ is the standard deviation of the Gaussian smoothing function.

In summary, we have shown that reasonable assumptions about the joint pair distribution functions in walls with non-zero thickness lead to a configuration factor that contains a "width factor" in addition to the "length factor" discussed in the previous subsection. For walls with sharp boundaries, this width factor has a $q_{t}^{-2}$ dependence in the large $q_{t}$ (Porod) regime. If the wall has a diffuse boundary, there is an additional $q_{t}$ dependence which can be quantified in terms of the smoothing function, $S(w)$. This additional $q_{t}$ dependence serves to increase the absolute magnitude of the slope of the scattering curve in $q$-space. The Gaussian case, which was worked out in detail, corresponds to a Gaussian smoothing function with a wall of zero width. All of these findings are in keeping with classical small-angle scattering. However, to reach these results, it was necessary to postulate zero correlation between the dislocations in the width direction. This may be a reasonable assumption in some cases, and where it breaks down drastically, it may be possible to revert to the dipole treatment of $\S I I$.

\section{Ordered and Partially Ordered Walls}

The long range interaction of the dislocations not only causes the dislocations to form wall structures, but also produces varying degrees of dislocation ordering within the walls themselves. Such internally-ordered walls scatter differently from disordered walls. In this subsection, some generic effects of such ordering within the walls will be explored.

Consider first the case of a totally ordered tilt wall, with $\mathrm{N}+1$ dislocations of the same sign, separated from one another by the distance, $L / N$,

$$
\begin{aligned}
\mathcal{W} & =b_{e}^{2} f^{2}(\xi)\left[\sum_{n=0}^{N} \exp \left\{i q_{w} n L / N\right\}\right]\left[\sum_{n=0}^{N} \exp \left\{-i q_{w} n L / N\right\}\right] \\
& =b_{e}^{2} f^{2}(\xi) \frac{\sin ^{2}\left(q_{w}(N+1) L / 2 N\right)}{\sin ^{2}\left(q_{w} L / 2 N\right)} \\
& \approx b_{e}^{2} N^{2} f^{2}(\xi) \frac{\sin ^{2}\left(q_{w} L / 2\right)}{q_{w}^{2} L^{2} / 4}, \quad\left(N \gg q_{w} L\right),
\end{aligned}
$$

which is the scattering function for a grating. For $q_{w} \ll N / L$, this result becomes indistinguishable from the case of a random wall with $B=N$, and $q_{w} \ll \sqrt{N / L^{2}}$. See Eqn. (27).

A completely ordered $B=0$ wall with alternating sign dislocations gives

$$
\begin{aligned}
\mathcal{W} & =b_{e}^{2} f^{2}(\xi)\left[\sum_{n=0}^{N}(-1)^{n} \exp \left\{i q_{w} n L / N\right\}\right]\left[\sum_{n=0}^{N}(-1)^{n} \exp \left\{-i q_{w} n L / N\right\}\right] \\
& \approx b_{e}^{2} f^{2}(\xi) \frac{\sin ^{2}\left(q_{w} L / 2\right)}{\cos ^{2}\left(q_{w} L / 2 N\right)} .
\end{aligned}
$$


Examination of Eqns. (39) and (40) shows that the effect of replacing every other positive dislocation with a negative dislocation is to "shift" the diffraction pattern by half the period, or $\pi N / L$. The reason for this unusual behavior is that each positive/negative dislocation pair forms a double-sized unit cell with peaks spaced half as far apart in reciprocal space. The multiplying form factor of the dislocation pair has zeros at the original lattice sites, causing the apparent shift. The width of the diffraction peak is unaffected. It is the presence of both positive and negative dislocations in the unit cell that distinguishes this case from the usual grating result. A somewhat different physical insight derives from the fact that the scattering from adjacent dislocations is phase shifted by $\pi$, so forward scattering is zero for $q_{w}=0$, unlike the usual situation where all elements of a structure scatter in phase.

It is necessary to avoid confusion regarding the periodicity in $q$-space associated with the wall of dislocations, which leads to a "grating function" for the dislocations, and the periodicity in the lattice which leads to the Bragg peaks. Because of the similarity in concept, we will use the term "dislocation Bragg" period to refer to the grating period of the ordered (and partially ordered) dislocation scattering.

In the literature on small-angle scattering from particles ${ }^{18,14,17}$, Debye has given a simple treatment of pair correlation which leads to analytic results, which is instructive to invoke for the dislocation case. In particular, if it is assumed that $\mathcal{P}(r)$ has the form

$$
\begin{aligned}
& \mathcal{P}(r)=0, \quad 0<r<l_{0}, \\
& \mathcal{P}(r)=1 / L^{2}, \quad l_{0}<r<L,
\end{aligned}
$$

then for such a distribution of simple dipoles or single-sign dislocations in a single slipsystem, Eqn. (20) takes the form

$$
\begin{aligned}
\langle\mathcal{W}\rangle & =b_{e}^{2} N f^{2}(\xi)+b_{e}^{2} N(N-1) f^{2}(\xi) \frac{2}{L^{2}} \int_{l_{0}}^{L}(L-r) \cos \left(q_{w} r\right) d r \\
& \approx b_{e}^{2} N f^{2}(\xi)+b_{e}^{2} N(N-1) f^{2}(\xi)\left[\frac{\sin ^{2}\left(q_{w} L / 2\right)}{q_{w}^{2} L^{2} / 4}-2\left(1-l_{0} / L\right) \frac{\sin \left(q_{w} l_{0}\right)}{q_{w} L}\right] .
\end{aligned}
$$

The first term in the brackets is the configuration factor for the random wall (see Eqn. (27)), and the second term is due to the lower cut-off at the exclusion distance, $l_{0}$. In the case of particles, the exclusion region exists because two particles cannot occupy the same space. In the case of dislocations, a similar result arises due to the strong repulsion between two dislocations of like sign at very small distances. For unlike sign, the dislocations will annihilate each other below a critical distance, even if spontaneous climb is necessary. This effect is observed as a maximum absolute density of dislocations in heavily deformed metals ${ }^{21}$. When $2 \mathrm{Nl}_{0} / L>1$, the configuration factor becomes negative outside the main peak, but this can never be the case, because it would mean that the mean distance between dislocations is smaller than the exclusion distance. For all physically-realistic parameters in Eqn. (42), the effect of a small exclusion zone around a dislocation is negligible.

Next, consider the important effect of partial ordering of defects in the wall. Rewriting Eqn. (22) for a single slip-system gives the symmetrical result

$$
\frac{\langle\mathcal{W}\rangle}{b_{e}^{2} f^{2}(\xi) N}=1-\frac{N^{+}}{N}\left\langle\mathcal{W}^{++}\right\rangle-\frac{N^{-}}{N}\left\langle\mathcal{W}^{--}\right\rangle
$$




$$
+\frac{N}{4}\left[\left(1+\frac{B}{N}\right)^{2}\left\langle\mathcal{W}^{++}\right\rangle+\left(1-\frac{B}{N}\right)^{2}\left\langle\mathcal{W}^{--}\right\rangle-2\left(1-\frac{B^{2}}{N^{2}}\right)\left\langle\mathcal{W}^{+-}\right\rangle\right]
$$

By symmetry, we can place two conditions on this equation

$$
\begin{aligned}
& \left.\left\langle\mathcal{W}^{++}\right\rangle\right|_{B=0}=\left.\left\langle\mathcal{W}^{--}\right\rangle\right|_{B=0} \\
& \left.\left\langle\mathcal{W}^{++}\right\rangle\right|_{B=N}=\left.\left\langle\mathcal{W}^{--}\right\rangle\right|_{B=-N}
\end{aligned}
$$

Also, the joint pair distribution function for partially-ordered dislocations is likely to resemble that for a partially-ordered two-component solid. Thus, for dislocations of the same sign, we consider the functional forms

$$
\begin{aligned}
& \mathcal{P}^{++}(r)=\frac{1}{L^{2}}\left(1+C e^{-r / \alpha} \cos ^{2}\left(\pi N^{+} r / L\right)\right), \\
& \mathcal{P}^{--}(r)=\frac{1}{L^{2}}\left(1+C e^{-r / \alpha} \cos ^{2}\left(\pi N^{-} r / L\right)\right),
\end{aligned}
$$

where $C$ is a parameter controlling the strength of the correlation and $\alpha$ is its range. For simplicity, we do not include an exclusion zone at the origin; such a zone would not substantially affect the wall configuration factor. Also, as long as $\alpha \ll L$, the error in the normalization for these equations will be negligible. The periods in these functions were chosen so that they would be correct when their contributions are significant. Thus, when $B=N$, the average spacing for the positive dislocations will be $N / L=N^{+} / L$, and when $B=0$, the average spacing will be $N / 2 L=N^{+} / L$. Eqn. (45) may become invalid for the minority population which will soon become very highly disordered, but the contribution of this term is minimized by the pre-factor $(1-B / N)^{2}$ in (43). Of course, in these equations, both $C$ and $\alpha$ should be functions of $B$. However, since we are only interested in qualitative behavior, we will let them be constants.

It remains to specify the correlation function for unlike pairs. The pre-factor for $\left\langle\mathcal{W}^{+-}\right\rangle$ has a maximum at $B=0$, making this the most important point for this term. Here, the energetics will favor pairing of unlike dislocations so that negative dislocations tend to sit between positives, etc. Thus, for simplicity, we take

$$
\mathcal{P}^{+-}(r)=\frac{1}{L^{2}}\left(1+C e^{-r / \alpha} \sin ^{2}(\pi N r / 2 L)\right)
$$

In reality, the correlation strength and range for unlike pairs will certainly not be the same as for like pairs, but such an approximation is adequate for our purpose of studying the qualitative features of partial ordering. As we move away from $B=0$, the period given in Eqn. (46) will remain correct because this is the smallest period possible for $(+-)$ pairs. Of course, the functional form may change once a single sign dominates, but then the pre-factor for $\left\langle\mathcal{W}^{+-}\right\rangle$becomes very small, once again minimizing any error.

Fig. 4 shows the results for this simplified version of partial order near $B=0$. Here, we plot only the $\left\langle\mathcal{W}_{m n}\right\rangle$ part of the total $\langle\mathcal{W}\rangle / b_{e}^{2} f^{2}(\xi) N$, i.e. the term proportional to $N / 4$ in Eqn. (43). Figs. $4(\mathrm{a})$ and $4(\mathrm{~b})$ use $B=0, N=20$, and $C=0.5$; the other parameters used are: (a) $\alpha=10, L=100$ and (b) $\alpha=20, L=200$. As expected, the position of the dislocation Bragg peak is set by the dislocation spacing, the width is determined by 
the correlation range, and the strength depends on the number of dislocations within this range. Thus, in Fig. 4(b), the dislocation Bragg peak shifts position and is narrower, both consistent with doubling the dislocation spacing. Figure 4(c) uses the same parameters as Fig. 4(a) except that $B=N / 10$, and shows the distinctive signature for the $B \neq 0$ case. The narrow peak at the origin is proportional to $B^{2}$ and it's width is proportional to the inverse of the wall length, $1 / L$. This is the same peak that is found for the ordered and random cases, Eqns. (39) and (27). That is, the peak at the origin is independent of order, and depends only on the nonzero value of $B$.

\section{COMPUTER SIMULATIONS.}

In this section, computer simulations are used to confirm the correctness of the analysis developed in the previous sections given the various assumptions that were made. Calculations are performed on a square X-Y lattice with unit spacing. This lattice does not represent the atomic positions in the sample; instead, we assume that a site of the lattice represents a region of underlying crystal. At each site, we use Eqn. (5) to compute the dilatation of a distribution of dislocations. Thus, the simulation averages the dilatation over a small region represented by the lattice site. The resulting sampling errors are minimized by considering only dislocation distributions that are sparse relative to the lattice spacing. Since the dilatation is singular at the core of a dislocation, we arbitrarily set the dilatation of a cell containing a dislocation center to zero. Eqns. (1) and (2) are then used to compute the scattered intensity directly from the dilatation of the lattice.

Three simulations are presented. In the first, the form factor of a single dislocation is examined. This is followed by a look at the configuration factor of a random wall of zero thickness with $B=N$. Finally, the case of a random wall of zero thickness with $B=0$ is investigated. The random wall results are obtained by using a random number generator to generate a large number of random dislocation configurations with the required parameters. The configuration factors calculated from these configurations are then averaged to obtain the final results. In all of the simulations, the Burgers vectors of the dislocations have equal magnitude and lie in the $X$-direction. Dislocation walls are aligned along the $Y$ axis, perpendicular to the slip plane. In this configuration, the $q_{w}$ direction is favorable for scattering, but no scattering will take place in the thickness direction because of the single dislocation form factor.

The first simulation is meant to confirm the scattering from a single dislocation as given by Eqn. (7). The dislocation is located at the center of a square lattice with an edge length of 400 ; the results are shown in Fig. 5. The units for $q_{w}$ are $2 \pi / d$, where $d$ is a length measured in units of lattice spacings. First, note that the scattered intensity goes to zero as $q_{w} \rightarrow 0$, and the expected oscillatory Bessel function character is confirmed. Also, the maximum of the scattering occurs at $q_{w}=2 \pi / R$ as predicted by Eqn. (7) with the upper cut-off distance, $R$, set to the distance from the dislocation to the sample edge. Finally, the log plot shown in Fig. 5(b) shows that the asymptotic form of the scattering is $1 / q_{w}^{2}$, for large $q_{w}$, as predicted. Not shown is the scattering for $q_{t}$, which is strictly zero, as predicted by Eqn. (7). Although the general behavior of the computer simulation matches the behavior predicted by Eqn. (7), we note that this agreement is not quantitative. The 
reason for the difference is that Eqn. (7) was derived with the assumption that finite-size effects are negligible. This is clearly not true for the computer simulations.

It is important to note that the oscillations predicted by Eqn. (7) and shown in Fig. 5 would not be observed in an actual experiment. This is because the coherence length, $R$, is not a sharp cut-off that is the same for all dislocations. Thus, averaging over the dislocations in a sample would produce an "averaged" structure factor with a simple $q_{w}^{-2}$ behavior and no oscillations.

It is useful to examine what happens to the scattering at $q_{w}=0$ when the dislocation is displaced from the origin in the $Y$-direction. This is related to the $q_{w}=0$ scattering for a dislocation dipole. When a single dislocation is displaced normal to its Burgers vector in the $Y$-direction, since the computation cell is finite, the net volume change in the cell is non-zero. This is because the number of atoms above the slip plane, where the dilatation is negative, is different from the number below the slip plane, where the dilatation is positive. Thus, the scattering at $q_{w}=0$ increases as the dislocation is displaced in the positive $Y$ direction. Similarly, for a dislocation dipole of positive and negative Burgers vector, with the dipole oriented along the $Y$-axis, there is again a net volume change for the computation cell, overall. Thus, a wall of alternating dislocations along the $Y$-axis will have a net volume change equivalent to a single dipole of effective displacement equal to the length of the wall and effective Burgers vector equal to $b / 2$ (or equivalently, a single whole dipole with a length equal to half the wall length).

The plots in Fig. 6 show simulations of the wall function, Eqn. (9), for randomized walls of zero thickness with 100 dislocations, which are averaged over 1000 independent iterations. In Fig. 6(a), all of the dislocations have the same sign and in Fig. 6(b), the dislocations have zero net Burgers vector. These simulations should be compared with Eqns. (27) and (26). In both cases, we have set $\left|b_{e}\right|=f(\xi)=1$. Fig. 6(a) for the same sign wall shows that the scattering has a strong peak at the origin proportional to the square of the number of dislocations. (Of course, the total scattered intensity is a product of the single dislocation function, which goes to zero at $q_{w}=0$, and otherwise multiplies the wall function by $q_{w}^{-2}$ when $q_{w}$ is large.) At large $q$, the function approaches it's asymptotic value of $N$. The $B=0$ wall function, depicted in Fig. 7(b), has a "hole" at the origin, and then rises to the same asymptotic value as before. In both Figs. 6(a) and 6(b) the peak (and hole) have widths given by $\delta q_{w}=2 \pi / L$, as predicted. We note that the wall function in both cases approaches an asymptotic value proportional to the total number of dislocations in the wall. This asymptotic value is the term we have called the "self scattering" term in Eqn. (12) and its later incarnations.

\section{CONCLUSIONS.}

The theory developed in this paper for small-angle scattering by dislocations in deformed single-crystals has the traditional feature that the scattering is a product of several independent factors. Aside from the atomic structure factor and the sample size form factor, neither of which is considered in this paper, the intensity is composed of a structure factor for the "single defect", which may be either a single dislocation or a dislocation dipole pair, and a configuration factor for the configuration of single defects. 
Experiments capable of measuring the relevant parameters will be difficult and very few existing facilities currently have the necessary equipment. Experimental difficulties include such requirements as avoidance of accidental Bragg peaks, adequate rotational control of the sample/beam geometry, high-quality single-crystal dog-bone shaped metal samples that are thin enough to limit multiple scattering, a tensile stage capable of deforming these samples in situ while simultaneously measuring the engineering stress and strain, high beam brightness, and ultra-low-angle capabilities. The smallest $q$ that can be reached using existing suitable USAXS facilities corresponds to a spatial distance of $\approx 1 \mu \mathrm{m}$. Thus, large structures such as entire dislocation walls and cells are not presently visible. However, the local organization of dislocations within a wall should certainly be accessible. Thus, the theory should be applied to the structure of walls, and to the scattering of individual dislocations, but not to the scattering by complete cells. A new USAXS facility under construction at the Advanced Photon Source will extend this usable $q$-range by about a factor of 5 , which should allow the observation of complete cells in many cases.

The structure factor for the single dislocation can be written as two separate factors corresponding to the " $q_{z}$ scattering" for the scattering due to the length coordinate of the dislocation, and the $q_{p}$-scattering normal to the length direction. The $q_{z}$-structure factor has a strong and narrow maximum when the scattering vector is normal to the length direction, with a simple "diffraction function" angular dependence. The scattered intensity reaches a maximum when the scattering vector is also perpendicular to the edge components of the Burgers vectors. For the single dislocation, this single defect angular variation is rather "soft" with the form $\sin ^{2} \xi$, while the dislocation dipole is more highly directional with a "spot light" in the direction normal to the slip plane of form $\sin ^{4} \xi$. Thus, a dislocation is best situated to scatter when the scattering vector is perpendicular to its slip plane.

The single dislocation structure factor is multiplied by the factor for the configuration of the dislocations, which we have assumed is a diffuse wall. The wall function, $\langle\mathcal{W}\rangle$, is composed of two additive terms. The first term is the self-scattering term which goes to zero as $q_{w} L \rightarrow 0$. In both of the specific cases examined (ie, the random case and the fully ordered case), this term goes to zero quadratically. For $q_{w} L \gg 1$, this first term in the wall function is independent of $q_{w}$, and is simply proportional to the total number of scattering dislocations (or dislocation dipoles). Because it is a constant for large $q_{w}$, this term in the wall function will be dominant when other contributions that decrease with $q_{w}$ have decayed. In the large $q_{w}$ domain, then, the scattering is characterized simply by the single defect structure factor, without the complication of the defect configuration, and the strength of the scattering is linear in the total number of scatterers. An important consequence of this fact is that if single dislocations and dipoles are both present in significant numbers, the problem becomes solvable in this $q_{w}$ range. Thus, the scattered intensity in this range will be the sum of $q_{w}^{-2}$ for single dislocations and $q_{w}^{-3}$ for dislocation dipoles. Experiments in this range could therefore settle the long-standing debate on the existence of dipoles in deformation structures. ${ }^{2,7,8}$

The second, $q$-dependent, term comprising the wall function is again composed of two separate factors that are multiplied together. The first factor describes the dimension along the wall (i.e. the $q_{w}$ direction), and perpendicular to the dislocation lines. (In the case of fcc crystals, it is reasonable to assume that the walls are composed of dislocations parallel to one another, but not necessarily on the same slip planes.) This dimension was normal to the 
slip plane in the computer simulations. This factor depends both on the net Burgers vector of the wall and on the presence of ordering in the $q_{w}$ direction. For a completely random distribution with $B=N$, this function has a peak at the origin proportional to $B^{2}$, which decreases quadratically to zero as a function of $q_{w}$ in the range $q_{w} L \gg 1$. For a completely random distribution with $B=0$, the term is zero.

When the dislocations are partially ordered, interference peaks appear associated with the inter-dislocation distance and the correlation length. For $B \neq 0$, the same peak at the origin appears which was discussed for the random case. For all $B$, a dislocation Bragg peak exists at a position characterized by the defect structure repeat distance. For complete ordering, the peak is sharp with a width given by the number of dislocations in the wall, but if the order is only short range, with correlations extending only a few inter-dislocation distances, then the peak is much broader.

The second factor in the $q$-dependent term describes the $q$-dependence normal to the wall in the thickness direction. The thickness configuration factor is a delta function when the thickness goes to zero, and otherwise depends on the distribution of the dislocations in the thickness direction. If the dislocations are distributed randomly within a thick wall, and the wall has a sharp interface, then the $q_{t}$ scattering will have a conventional $q_{t}^{-2}$ behavior. If the interface is diffuse rather than sharp, the power will deviate from -2 to $-(2+\delta)$. $\delta$ contains information on the width of the diffuse boundary.

In summary, any experimental investigation of the dislocation structures must be guided by geometrical considerations of the incident X-ray or neutron direction, and its relation to the relevant slip systems of the material. It is found that only those dislocations lying normal to the scattering vector will contribute significantly to the scattering due to the strong peak in the configuration factor for the single dislocation. Further, the scattering will be peaked (very strongly in the case of dislocation dipoles) for $\overrightarrow{\mathbf{q}}$ also perpendicular to the Burgers vector. Thus, the most favorable scattering geometry will be one with the scattering vector perpendicular to the slip plane of the dominant dislocations. Above a particular $|\overrightarrow{\mathbf{q}}|$, the scattering will be sensitive only to the total number of edge dislocations, while below this value, the scattering will be sensitive to the ordering within the walls. For carpet structures, the scattering will be determined by the distribution in the thickness direction, while for walls normal to the dominant slip plane, the scattering will be determined by the ordering within the plane of the walls and the net Burgers content. Structural features will be accessible only to the extent that the lowest measurable $|\vec{q}|$ corresponds to the length scale of the relevant structure. Thus, dislocation structure within a wall should be readily accessible by current USAXS, while the cellular structure formed by the walls will be accessible only by the next generation facilities and for specimens at relatively high strain levels. In theory, it should be possible to obtain considerable information about dislocation structures in a deformed metal by a considered variation of the geometry between the incident beam and the active slip systems of the material. It remains to be seen just how much information can be extracted from such experiments in practice. This question is being pursued further. 


\section{REFERENCES}

${ }^{1}$ L. E. Levine and G. G. Long, to be published.

${ }^{2}$ D. Kuhlmann-Wilsdorf, Phys. Stat. Sol (a), 149, 131 (1995).

${ }^{3}$ A. Argon, in Physical Metallurgy, ed. R. W. Cahn and P. Haasen, 3, p, 1878, North Holland, (1996) New York.

${ }^{4}$ U. F. Kochs, in Proceedings of 50th Anniversary Symposium on Dislocations and Properties of Real Materials, The Institute of Metals, London, p 125 (1985).

${ }^{5}$ J. W. Steeds, Proc. Roy. Soc., A292, 343 (1966).

${ }^{6}$ A. D. Rollett, Strain Hardening at Large Strains in Aluminum Alloys, LA-11202-T (Los Alamos Natl. Laboratory, NM).

${ }^{7}$ H. Mughrabi, T. Ungar, W. Kienle, and M. Wilkens, Phil. Mag., 53, 793 (1986).

${ }^{8}$ A. Argon and P. Haasen, Acta Metall. et Mater., 41, 3289 (1993).

${ }^{9}$ A. Seeger and E. Kroner, Z. Naturforschg., 14a, 74 (1959).

${ }^{10}$ A. Seeger, J. Appl. Phys., 30, 629 (1959).

${ }^{11}$ A. Seeger and P. Brand., Small Angle Scattering, H. Brumberger, Ed., Gordon and Breach, NY, (1965).

${ }^{12}$ H. H. Atkinson and P. B. Hirsch, Phil. Mag., 3, 476 (1958).

${ }^{13}$ L. E. Levine and Robb Thomson, Acta Cryst., A53, 590 (1997).

${ }^{14}$ A. Guinier, X-Ray Diffraction in Crystals, Imperfect Crystals, and Amorphous Bodies, Dover, New York, (1994).

${ }^{15}$ J. P. Hirth and J. Lothe, "Theory of Dislocations", Wiley, New York, 1982.

${ }^{16}$ D. Dexter, Phys. Rev., 90, 1007 (1953).

${ }^{17}$ G. Porod, in Small Angle X-Ray Scattering, ed. O. Glatter and O. Kratky, Academic Press (1982) New York.

${ }^{18}$ P. Debye, Phys. Z., 31, 348 (1930).

${ }^{19}$ J. T. Koberstein, B. Morra and R. S. Stein, J. Appl. Cryst., 13, 34 (1979).

${ }^{20} \mathrm{~W}$. Ruland, J. Appl. Cryst., 4, 70 (1971).

${ }^{21}$ U. Essman and H. Mughrabi, Phil. Mag. A40 731 (1979). 


\section{FIGURES}

FIG. 1. Coordinate system for dislocation. The dislocation lies along the Z-axis perpendicular to the paper at the origin, and the edge component of the Burgers vector lies along the $\mathrm{X}$-axis. The projection of the scattering vector, $\overrightarrow{\mathbf{q}}$, in the X-Y plane has cylindrical coordinates $\left(q_{p}, \xi\right)$. A point in the X-Y plane has the cylindrical coordinates, $(r, \theta)$. The wall extends in the $\overrightarrow{\mathrm{w}}$ direction with $\vec{t}$ describing the perpendicular direction.

FIG. 2. A dislocation dipole at the origin is oriented along the $\mathrm{Y}$-axis, with separation distance $2 d$. The edge component of the Burgers vectors of the dipole is along the $\mathrm{X}$-axis. The coordinate definitions are the same as those in Fig. 1.

FIG. 3. Coordinate system for integration over wall variables. In (a), the original integration region in the variables $\left(R, R^{\prime}\right)$ is a square of side $L$. In the new variables, $\left(r, r^{\prime}\right)$, the integration region is the rotated rhombus, (b).

FIG. 4. Partially ordered wall. (a) Plot of term proportional to $N / 4$ in Eqn. (42) with $L=100$, $C=0.5, \alpha=10, N=20$, and $B=0$. The plot shows the dislocation Bragg peak with the position set by the dislocation spacing, the width set by the correlation distance, and the strength by the number of correlated dislocations. (b) Same as above, with $L=200$ and $\alpha=20$. The peak has shifted position to half its previous $q_{w}$-value. Its peak height is the same, because the same number of correlated dislocations are present in each case and so is the total number of dislocations in the wall. The peak width is reduced by half, because the correlation distance is increased. (c) Same as (a), with $B / N=0.1$. Now a small narrow peak appears at the origin. As $B$ is increased, this peak grows proportionally to $B^{2}$.

FIG. 5. Scattering for a single dislocation at the origin of a square lattice with 401 sites on a side. a) is a cut along the $q_{w}$-axis, showing the intensity of scattering as a function of $q$. b) is a log plot of the same data showing that the $q_{w}$ dependence for large $q_{w}$ is $q_{w}^{-2}$.

FIG. 6. Wall configuration factors for random walls containing 100 dislocations with (a) $B=N$ and (b) $B=0$. The figures show $\langle\mathcal{W}\rangle$ plotted against $q_{w}$. The wall length is 500 lattice spacings. Dislocation positions are randomized and averaged over 1000 simulations. In both cases, the asymptotic value for large $q_{w}$ is the total number of dislocations. 



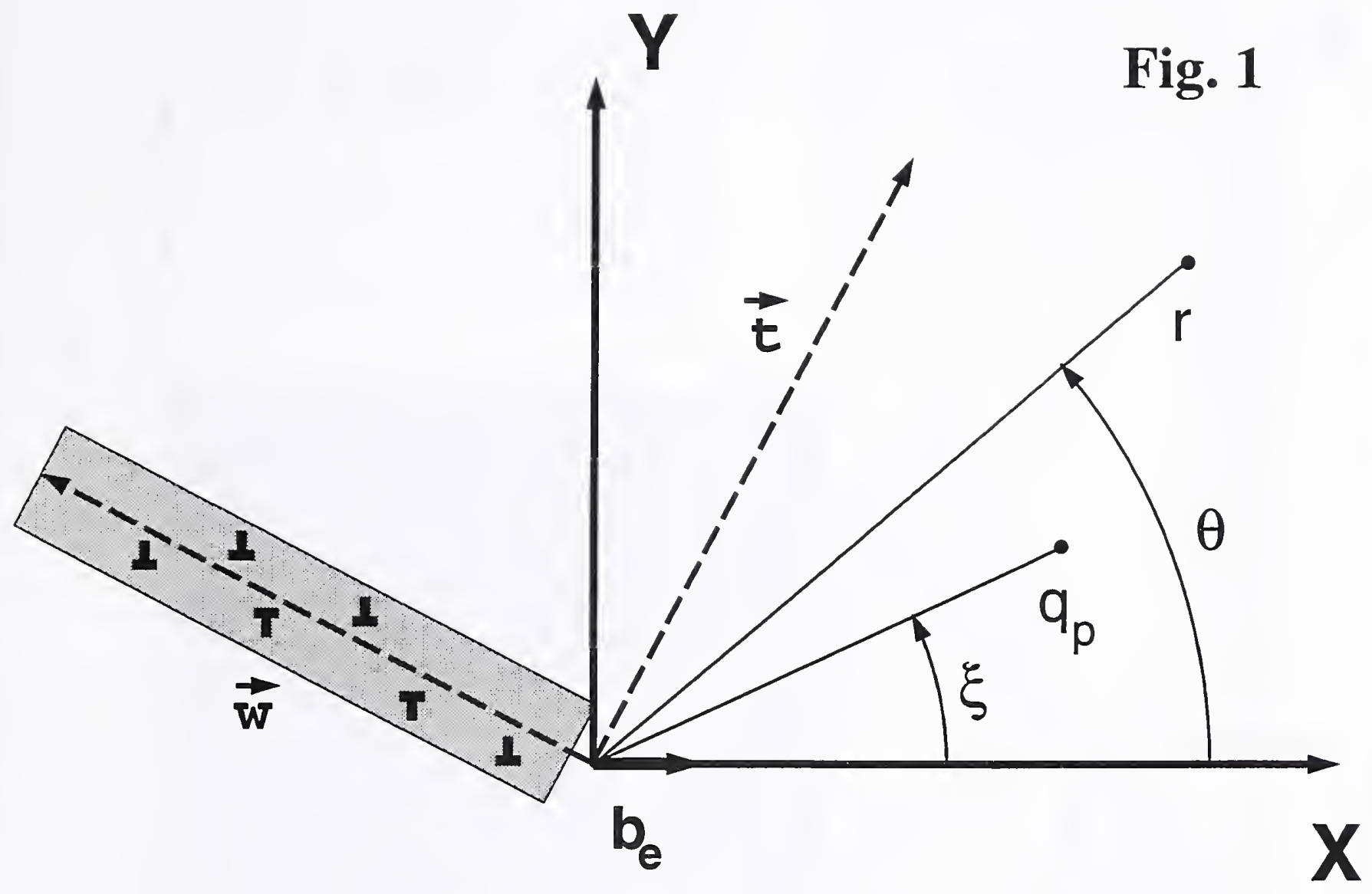




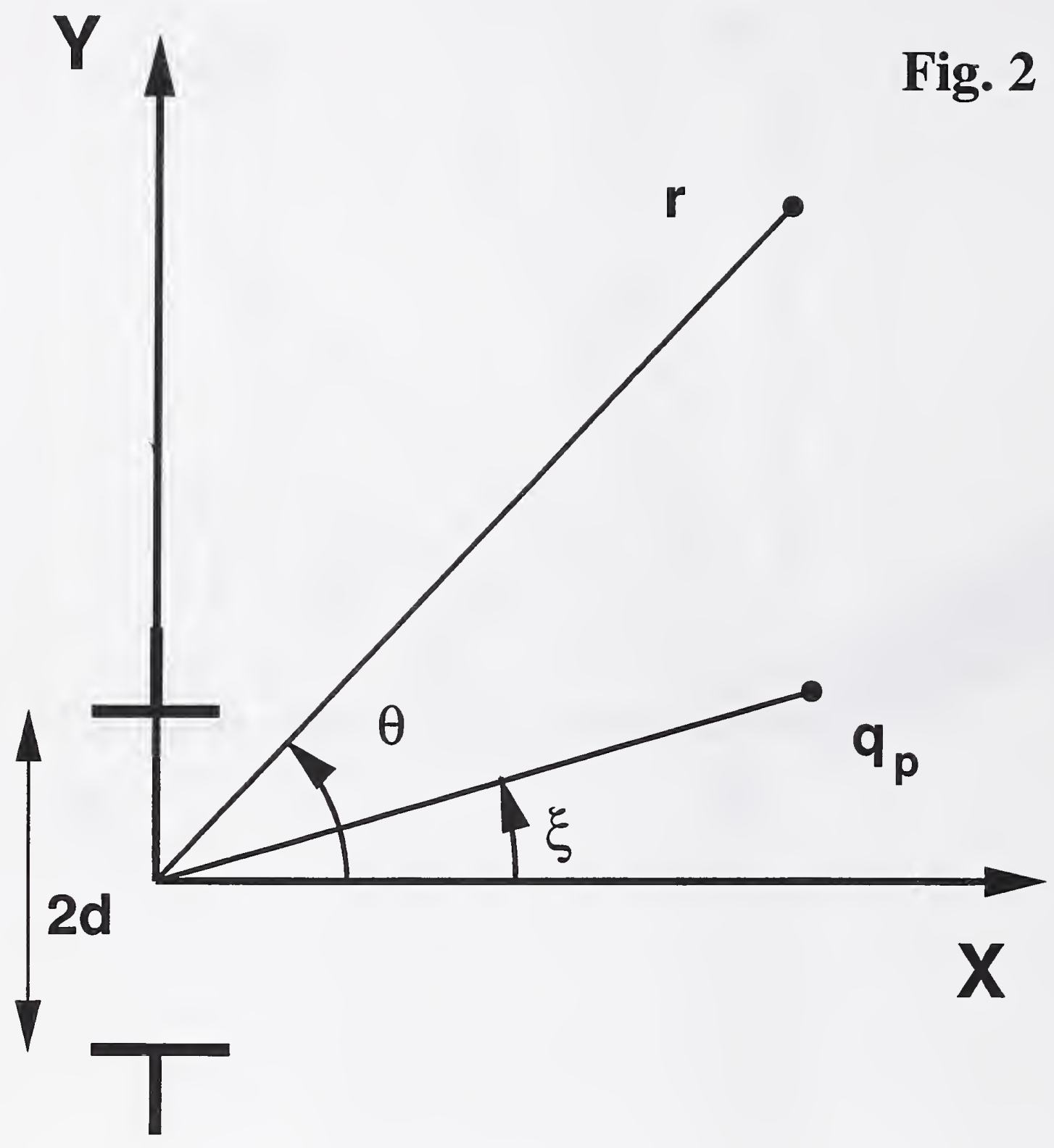




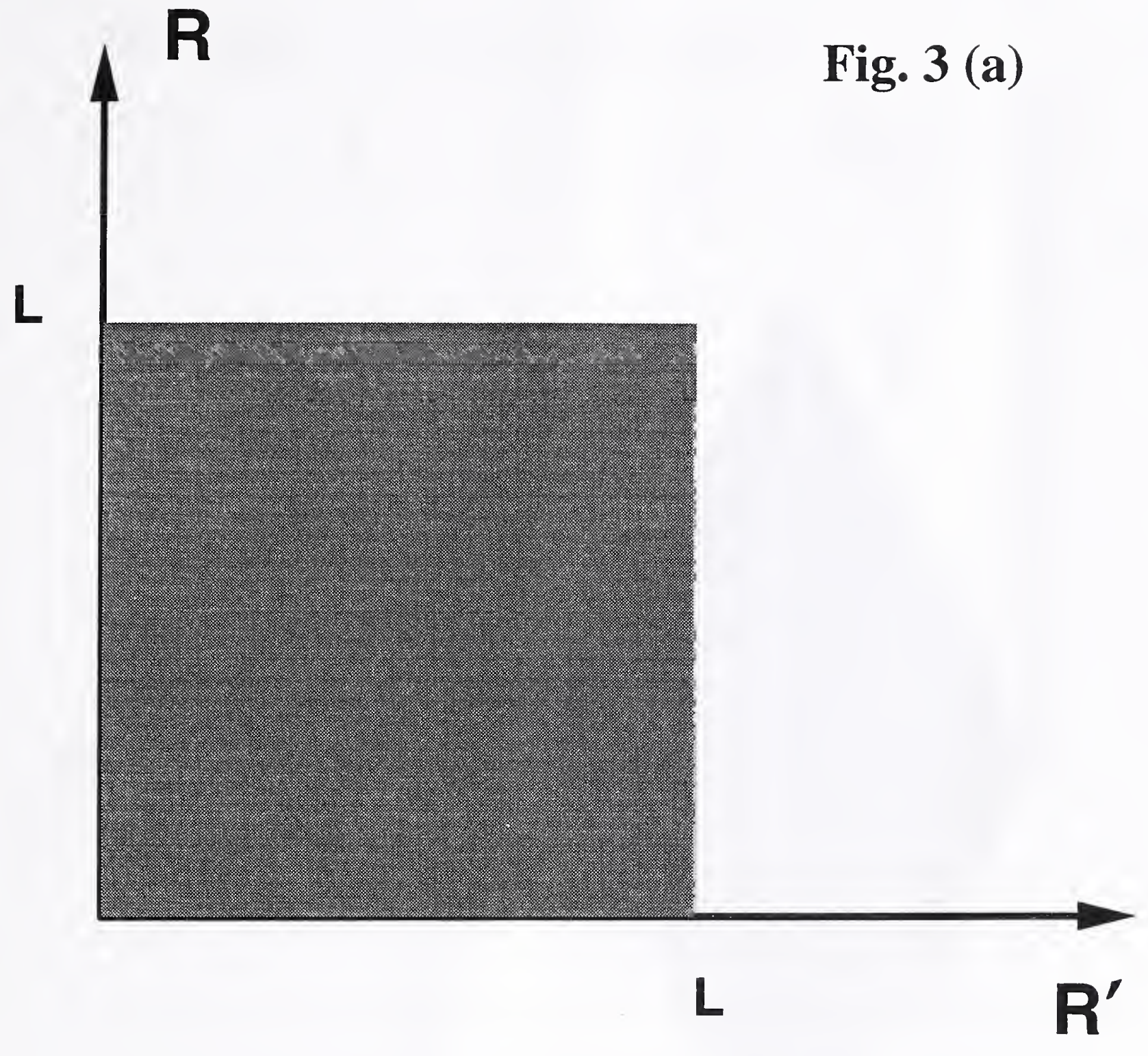




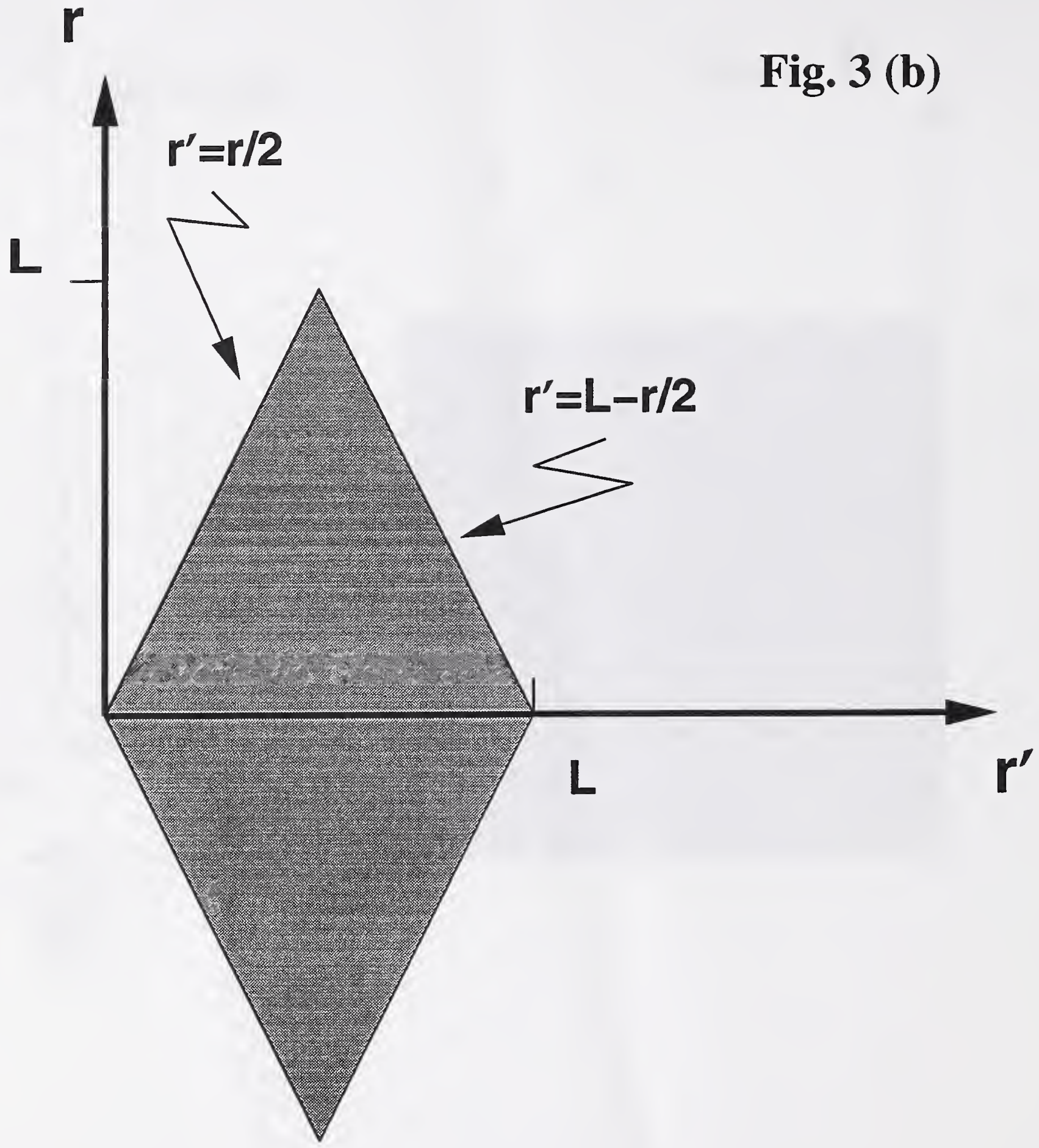




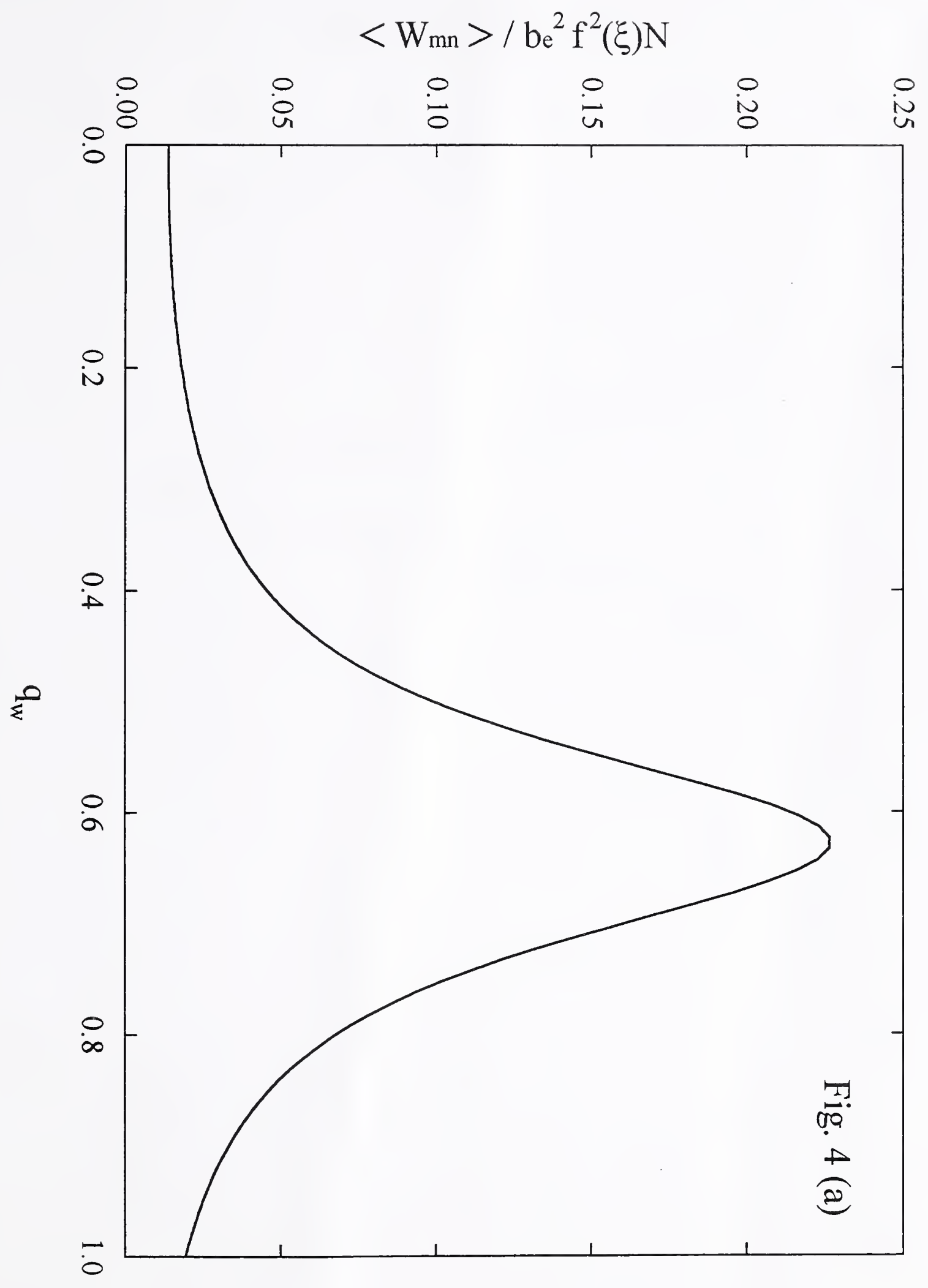




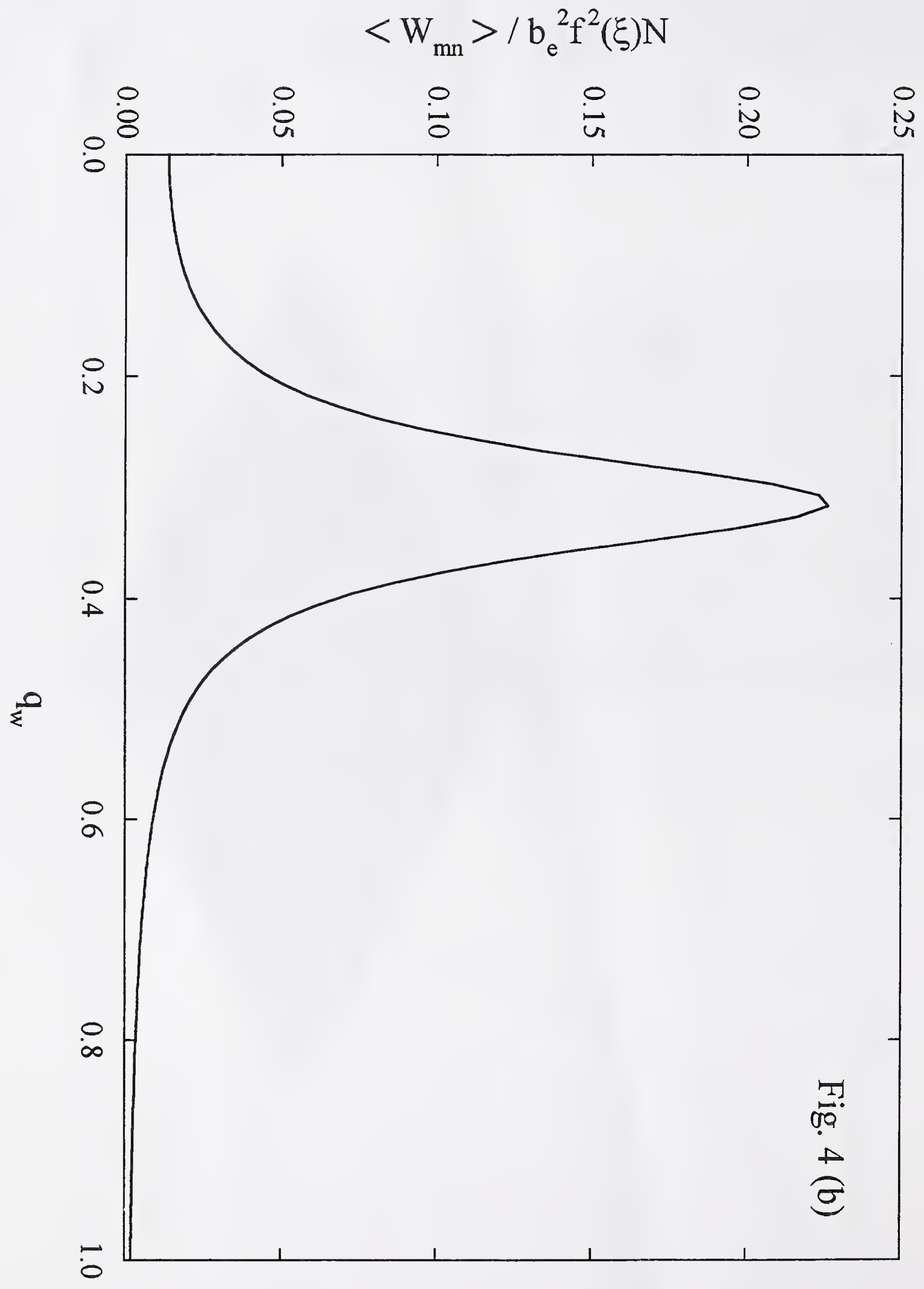




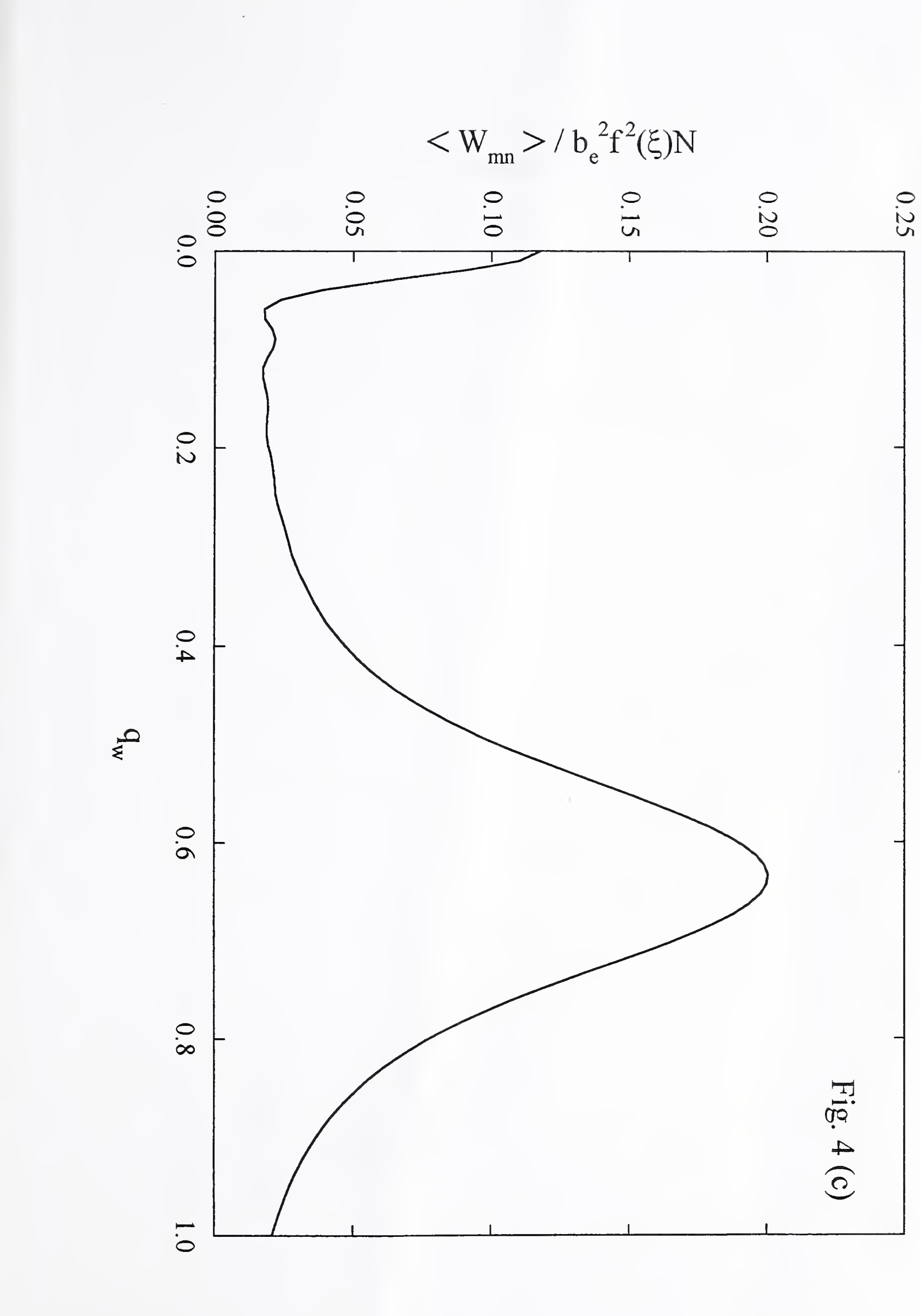




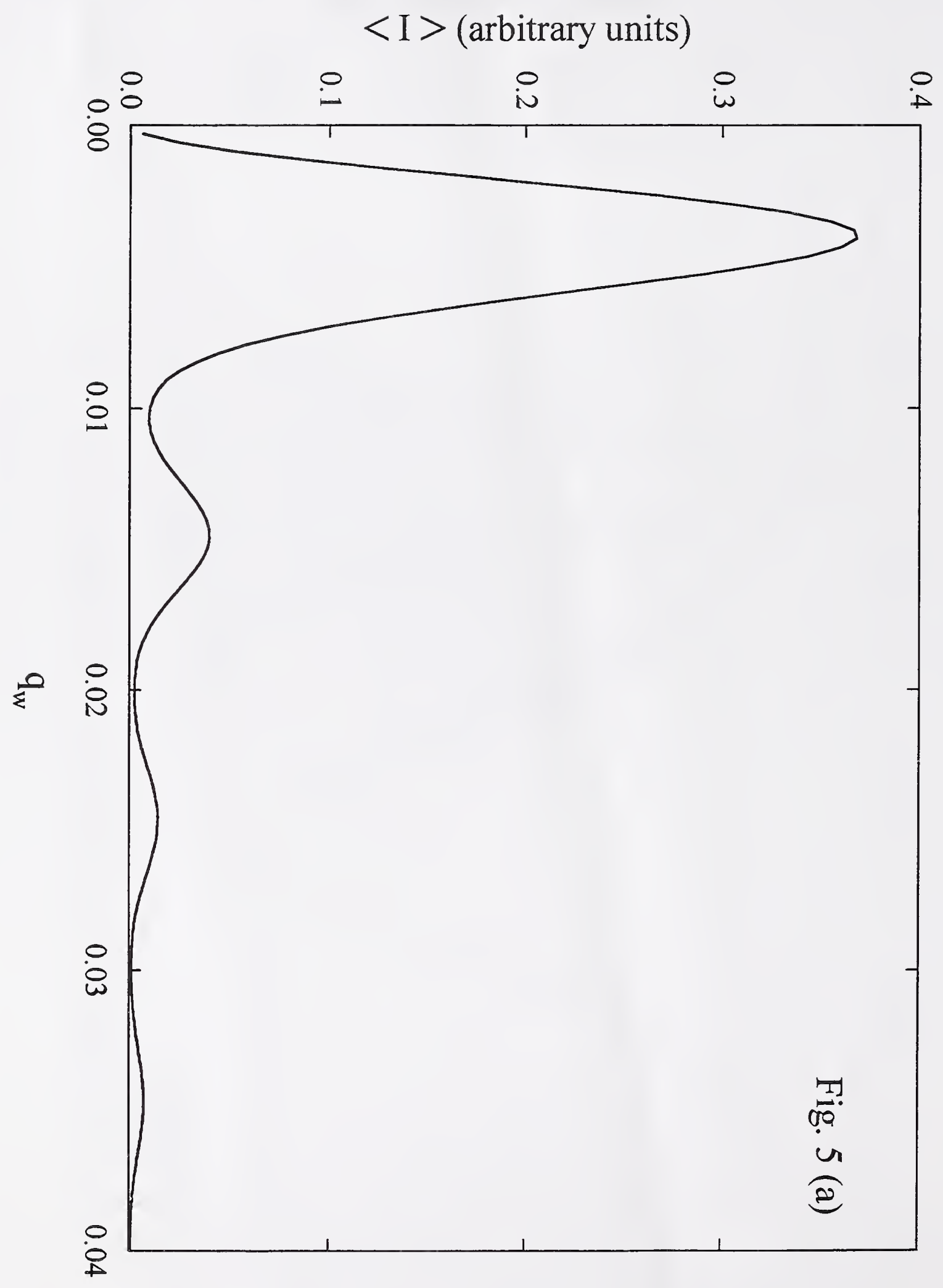




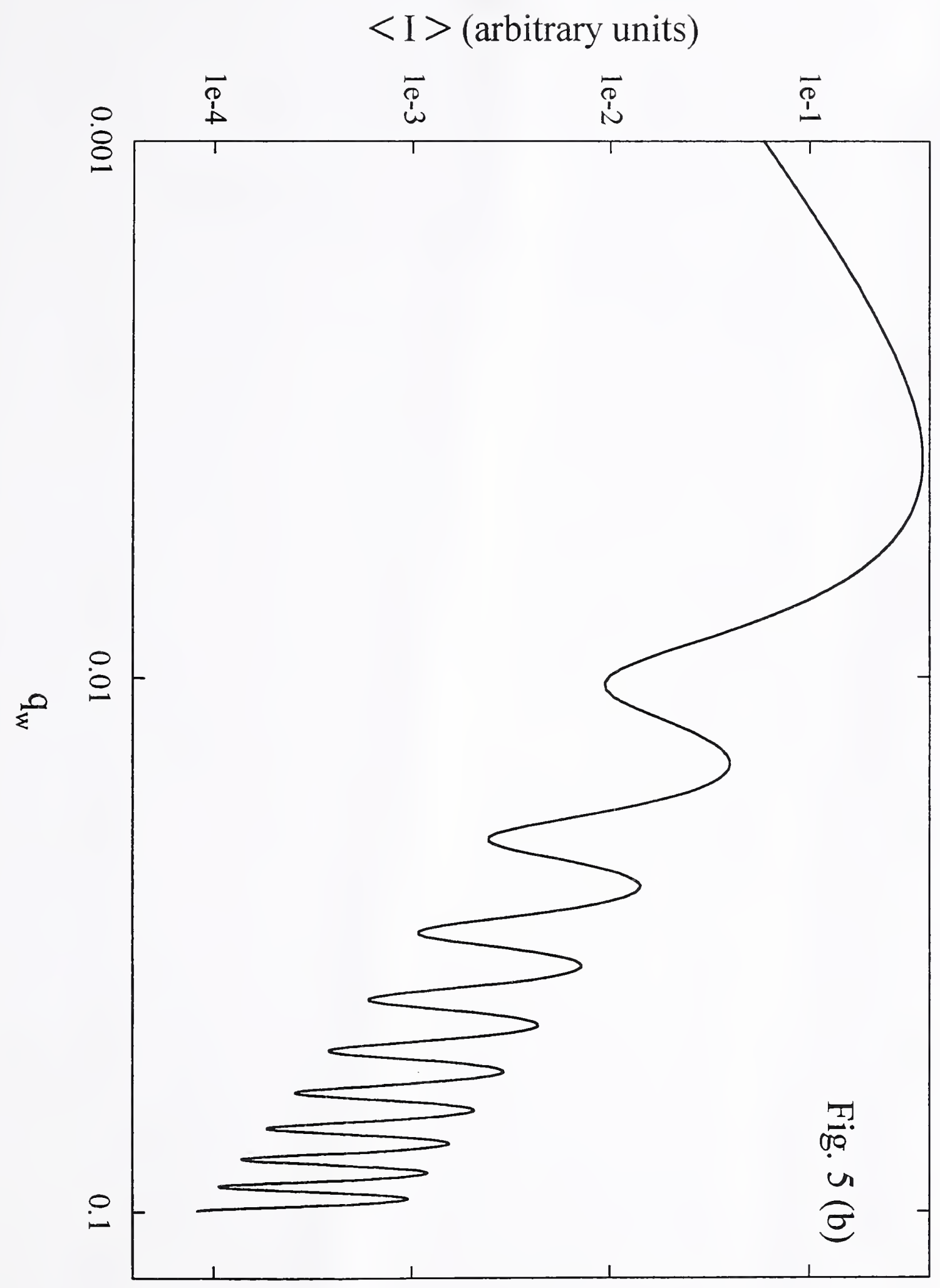




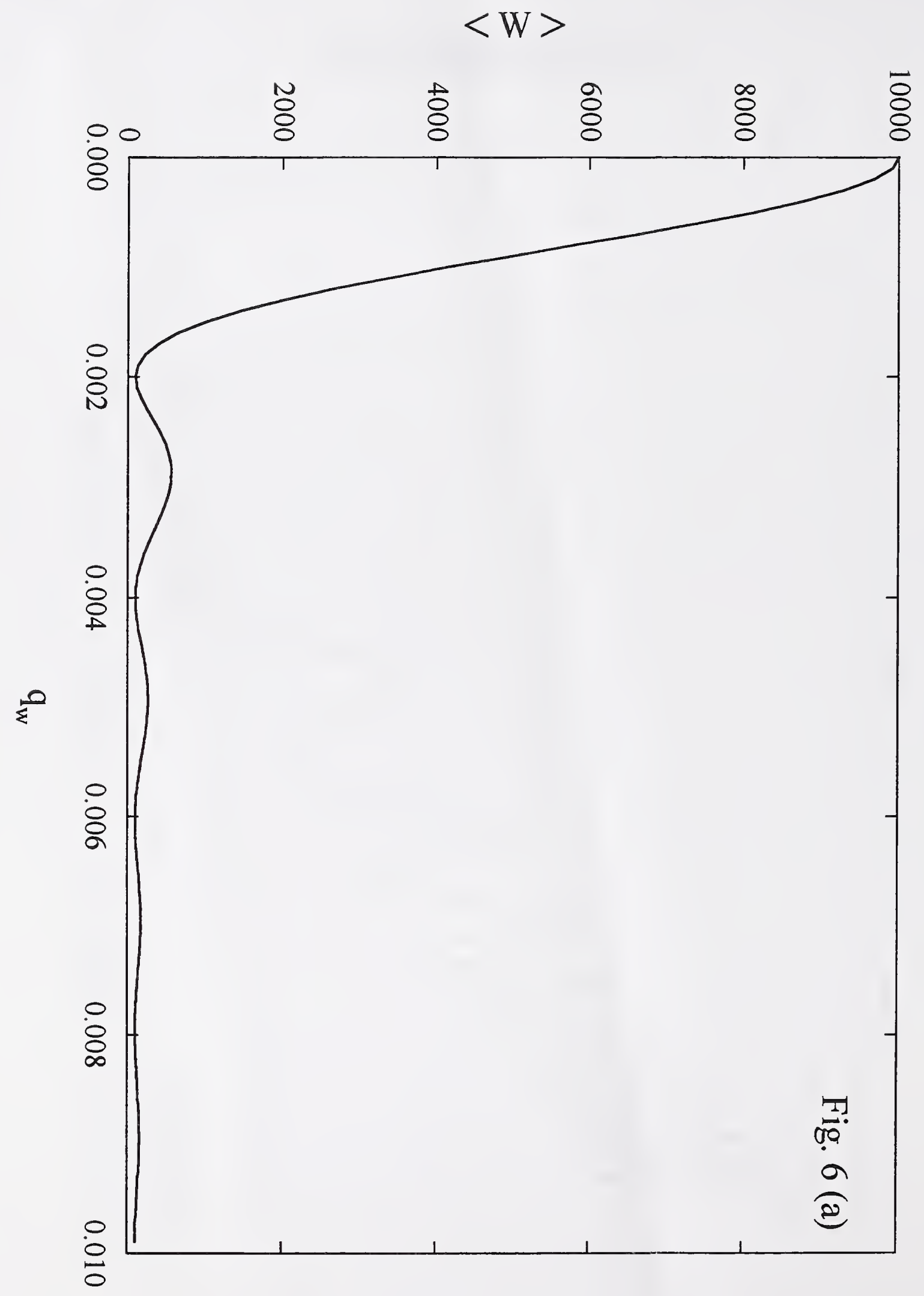




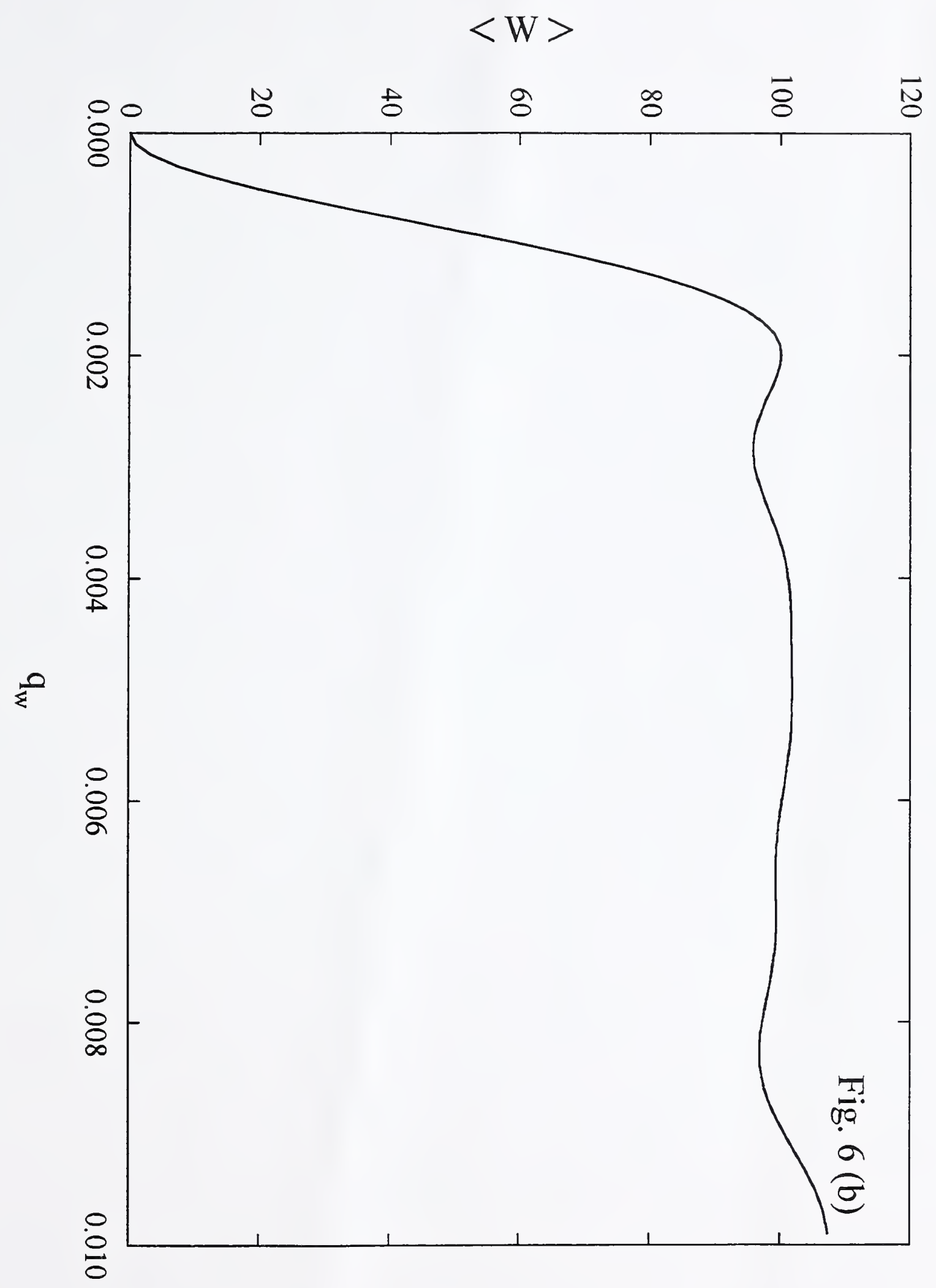



